\title{
PARITY SHEAVES, MOMENT GRAPHS AND THE $p$-SMOOTH LOCUS OF SCHUBERT VARIETIES
}

\author{
PETER FIEBIG AND GEORDIE WILLIAMSON
}

\begin{abstract}
We show that, with coefficients in a field or complete local principal ideal domain $k$, the Braden-MacPherson algorithm computes the stalks of parity sheaves with coefficients in $k$. As a consequence we deduce that the Braden-MacPherson algorithm may be used to calculate the characters of tilting modules for algebraic groups and show that the $p$-smooth locus of a (Kac-Moody) Schubert variety coincides with the rationally smooth locus, if the underlying Bruhat graph satisfies a GKM-condition.
\end{abstract}

\section{Contents}

1. Introduction 2

1.1. Acknowledgements 6

2. Equivariant sheaves 6

2.1. The equivariant derived category of a $G$-space 7

2.2. The equivariant functor formalism $\quad 7$

2.3. Equivariant cohomology 8

2.4. The Mayer-Vietoris sequence 9

2.5. The case of a torus 9

2.6. An attractive proposition 9

3. The localisation homomorphism 11

3.1. One-dimensional orbits 12

3.2. The localisation theorem - part I 12

4. The image of the localisation homomorphism 16

4.1. Sheaves on moment graphs 17

4.2. Sections of sheaves and the structure algebra 18

4.3. The costalks of a sheaf 18

4.4. The moment graph associated to a $T$-variety 18

4.5. The functor $\mathbb{W} \quad 19$

4.6. The case $X=\mathbb{P}^{1} \quad 19$

4.7. The localisation theorem - part II 19

5. Equivariant parity sheaves 21

5.1. Stratified varieties 22

5.2. Equivariant parity sheaves 22 
5.3. Short exact sequences involving parity sheaves 23

5.4. Further properties of equivariant parity sheaves 24

5.5. Obtaining parity sheaves via resolutions 25

5.6. Parity sheaves and the functor $\mathbb{W} \quad 27$

6. Braden-MacPherson sheaves on a moment graph 29

6.1. Extending local sections $\quad 29$

6.2. Braden-MacPherson sheaves 31

6.3. Directed moment graphs from stratified varieties 33

6.4. The $k$-smooth locus of a moment graph 34

6.5. The combinatorics of parity sheaves 35

7. The case of Schubert varieties 35

8. $p$-Smoothness 37

8.1. Smoothness and $p$-smoothness 38

8.2. $k$-smoothness and the intersection cohomology complex $\quad 39$

8.3. $k$-smoothness and stalks 39

8.4. On the $p$-smooth locus of $T$-varieties $\quad 41$

8.5. A freeness result 42

9. Representations of reductive algebraic groups 43

9.1. Tilting modules 44

9.2. Simple rational characters 44

References $\quad 45$

\section{IntRODUCTION}

In Lie theory, one of the most successful methods to calculate representation theoretic data (such as character formulae, decomposition numbers or Jordan-Hölder multiplicities) is to find a geometric or topological interpretation of the problem. In many examples one obtains representation theoretic information from the stalks of intersection cohomology complexes on an associated algebraic variety (for example the flag variety, the nilpotent cone, or the group itself).

In the most successful applications of this approach (the KazhdanLusztig conjecture, canonical bases, character sheaves ...) the representation theoretic objects under consideration are assumed to be defined over a field of characteristic 0 . In this case the decomposition theorem often allows one to recursively calculate the stalks of intersection cohomology complexes, hence solving (or at least providing a combinatorial algorithm to solve) the representation theoretic problem.

However, recently a number of authors have pointed out that geometry also has something to say about modular representation theory 
(see [JMW09b] for a survey). In this article we are motivated by the following two examples of this phenomenon:

- For a ring $k$, the geometric Satake equivalence (cf. [MV07]) provides an equivalence of tensor categories between equivariant perverse $k$-sheaves on the affine Grassmannian and rational representations of the Langlands dual group scheme over $k$.

- In [Fie07b] and [Fie07a] a certain category of sheaves of $k$-vector spaces on an affine flag variety was related to representations of the $k$-Lie algebra associated to the Langlands dual root system. Here $k$ is assume to be a field whose characteristic is required to be at least the associated Coxeter number. The relation is then used to give a new proof of Lusztig's conjecture on the simple rational characters for reductive groups in almost all characteristics.

In [JMW09a] (motivated by ideas of Soergel [Soe00] together with a desire to better understand such relationships) a new class of sheaves, the "parity sheaves", was introduced. These are certain constructible sheaves on a stratified algebraic variety, which satisfy a parity vanishing condition with respect to stalks and costalks. It was shown that, under some additional assumptions, the indecomposable parity sheaves are parametrized in the same way as the intersection cohomology complexes. If the coefficients are of characteristic 0 the decomposition theorem often implies that the indecomposable parity sheaves are isomorphic to intersection cohomology complexes (up to a shift).

In positive characteristics this needs no longer be true. However, with coefficients of positive characteristic parity sheaves are often easier to work with than intersection cohomology complexes. Moreover, for some representation theoretic applications they may even form their natural replacement. For example,

- the category considered in [Fie07b, Fie07a] turns out to be the category of parity sheaves,

- under the geometric Satake equivalence (and under some mild and explicit assumptions on the characteristic of $k$ ) the parity sheaves correspond to tilting modules for the Langlands dual group (cf. [JMW09b]).

In the above results, fundamental representation theoretic data is encoded in the stalks of the indecomposable parity sheaves. It is therefore an important problem to find an algorithm for their calculation.

For an appropriately stratified complex algebraic variety $X$ with torus action Braden and MacPherson [BM01] describe an algorithm for 
calculating the stalks of intersection cohomology complexes with coefficients in a field of characteristic 0 (using localisation techniques in equivariant cohomology developed by Goresky, Kottwitz and MacPherson [GKM98]). The torus action has, by assumption, only finitely many fixed points and one-dimensional orbits. The structure of the oneskeleton of the torus action can be encoded in the "moment graph" of the variety:

- the vertices and edges are given by the torus fixed points and one-dimensional orbits respectively, with a one-dimensional orbit incident to those fixed points in its closure,

- each edge is labelled by a character of the torus determining an isomorphism of the orbit with $\mathbb{C}^{*}$ (this is defined only up to a sign).

Braden and MacPherson then describe an algorithm (using only commutative algebra) to produce a "sheaf" on the moment graph, and show that its stalks agree with those of the equivariant intersection cohomology complex. Thus the (a priori extremely difficult) computation of the stalks of the intersection cohomology complex may (in principle) be carried out in an elementary way.

Now, the Braden-MacPherson algorithm makes sense with coefficients in an arbitrary field $k$, or even in a local ring. However, simple examples show that it does not compute the stalks of intersection cohomology complexes when the coefficients are not of characteristic 0 . The central result of this paper is the following:

Theorem 1.1. Suppose that the pair $(X, k)$ satisfies the GKM-condition (cf. Section 4.7). Then the Braden-MacPherson algorithm computes the stalks of indecomposable parity sheaves.

In the theorem, $k$ denotes a complete local principal ideal domain. If $k$ is a field, then the GKM-condition may be stated simply: one requires that, for all pairs of one-dimensional orbits having a common torus fixed point in their closure, the corresponding characters do not become linearly dependent modulo $k$. This condition can easily be read off the associated moment graph.

In the course of the proof of the above result we provide a version of localisation theorem of [GKM98] with coefficients in a ring, i.e. we show that the hypercohomologies of certain equivariant sheaves on $X$ are given by the global sections of associated moment graph sheaves (see Theorem 4.4). For complete local rings we then show that the BradenMacPherson algorithm yields the objects associated to parity sheaves (see Theorem 6.10). In contrast to the proof of Braden-MacPherson, 
our arguments are more elementary, as we do not need the theory of mixed Hodge modules. As in characteristic 0 the decomposition theorem implies that the parity sheaves are intersection cohomology complexes up to a shift, we obtain a new proof of their result.

Applying the above theorem to the affine Grassmannian and using the Satake equivalence, we obtain:

Theorem 1.2. Suppose that $p>h+1$, where $h$ denotes the Coxeter number of our datum. On the moment graph of the affine Grassmannian and with coefficients in the ring of p-adic integers, the BradenMacPherson algorithm calculates the characters of tilting modules of the Langlands dual group over $\overline{\mathbb{F}}_{p}$.

The moment graph of the affine Grassmannian is GKM for a field $k$ if and only if $k$ is of characteristic 0 . We avoid this complication by using the $p$-adic integers in the above theorem.

We apply the multiplicity one result of [Fie10] to obtain a description of the $p$-smooth locus of Schubert varieties. Recall that an $n$ dimensional algebraic variety $X$ is $p$-smooth if for all $x \in X$ one has an isomorphism of graded vector spaces

$$
H^{\bullet}\left(X, X \backslash\{x\}, \mathbb{F}_{p}\right) \cong H^{\bullet}\left(\mathbb{C}^{n}, \mathbb{C}^{n} \backslash\{0\}, \mathbb{F}_{p}\right) .
$$

The $p$-smooth locus of $X$ is the largest open $p$-smooth subvariety. One similarly defines rationally smooth, and the rationally smooth locus by replacing $\mathbb{F}_{p}$ by $\mathbb{Q}$ above. If $X$ is rationally (resp. $p-$ ) smooth it satisfies Poincaré duality with rational (resp. $\mathbb{F}_{p^{-}}$) coefficients. Here is our result:

Theorem 1.3. Let $\mathcal{G}$ be the moment graph of a (Kac-Moody) Schubert variety $X$ and suppose that $\left(\mathcal{G}, \mathbb{F}_{p}\right)$ is a $G K M$-pair. Then the p-smooth locus of $X$ coincides with its rationally smooth locus.

In the finite dimensional case, the GKM-condition is always satisfied if $p \neq 2$ and if, in addition, $p \neq 3$ in $G_{2}$. This answers a (stronger version of) a question of Soergel (cf. [Soe00]). In fact, we prove the above theorem for a larger class of varieties with an appropriate torus action for fields $k$ that satisfy the GKM-condition.

The smooth and rationally smooth locus of Schubert varieties has been the subject of much investigation by a number of authors. See for example [Car94], [Kum96], [Dye93], [Dye05] and [Ara98]. It a result known as Peterson's theorem that the smooth and rationally smooth locus agree in simply-laced type, which immediately implies the above theorem. However, there are examples in non-simply-laced types of 
small rank where the 2-smooth and 3-smooth locus do not agree with the rationally smooth locus.

Lastly let us remark that results of this paper (in particular Section 8.5 ) are used in an essential way in the paper [JW], which shows that that Kumar's criterion for the rational smoothness of Schubert varieties can be extended to provide a criterion for $p$-smoothness. In particular, the main result of $[\mathrm{JW}]$ provides a means to determine the $p$-smooth locus when the underlying moment graph is not GKM, complementing Theorem 1.3. On may also use Theorem 1.3 together with the results of [JW] to obtain a novel proof of Peterson's theorem.

1.1. Acknowledgements. We would like to thank Daniel Juteau and Olaf Schnürer for useful conversations and Michel Brion for useful correspondence. P.F. gratefully acknowledges the support of the Landesstiftung Baden-Württemberg as well as the DFG-Schwerpunkt 1388 "Representation Theory". Both authors gratefully acknowledge the support of the Newton Institute in Cambridge, where parts of this paper were written.

\section{EquiVARIANT SHEAVES}

In this section we recall the construction of the bounded equivariant derived category $D_{G}^{b}(X, k)$ that is associated to a topological group $G$, a ring of coefficients $k$ and a $G$-space $X$. To a suitable continuous $G$-equivariant map $f: X \rightarrow Y$ one associates the push-forward functors

$$
f_{*}, f_{!}: D_{G}^{b}(X, k) \rightarrow D_{G}^{b}(Y, k)
$$

and the pull-back functors

$$
f^{*}, f^{!}: D_{G}^{b}(Y, k) \rightarrow D_{G}^{b}(X, k)
$$

satisfying a Grothendieck formalism. We then recall the equivariant cohomology $\mathbb{H}_{\mathrm{G}}^{\bullet}(\mathcal{F})$ of $X$ with coefficients in $\mathcal{F} \in D_{G}^{b}(X, k)$ and, finally, the Mayer-Vietoris sequence associated to an open $G$-stable covering $X=U \cup V$.

We will be mainly concerned with the following situation: $G$ will either be a complex algebraic torus, i.e. $G \cong\left(\mathbb{C}^{\times}\right)^{r}$ for some $r>0$, endowed with its metric topology, or its compact subtorus $\left(S^{1}\right)^{r}$. The space $X$ will be a complex algebraic variety with an algebraic $G$-action, and endowed with its metric topology. The main reference for the following is [BL94]. 
2.1. The equivariant derived category of a $G$-space. We fix a topological group $G$. A $G$-space is a topological space endowed with a continuous $G$-action. There always exists a contractible $G$-space with a topologically free $G$-action. We fix one of those and call it $E G$. For any $G$-space $X$ we can now define the quotient $X_{G}:=X \times_{G} E G$ of $X \times E G$ by the diagonal $G$-action. Then we have two maps

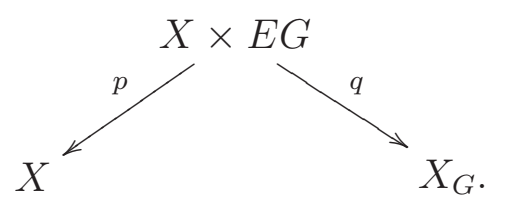

The map $q$ on the right is the canonical quotient map and $p$ is the projection onto the first factor.

Now we fix a ring of coefficients $k$. For any topological space $Y$ we denote by $D(Y, k)$ the derived category of sheaves of $k$-modules on $Y$. By $D^{b}(Y, k)$ we denote the full subcategory of objects with bounded cohomology. For a continuous map $f: Y \rightarrow Y^{\prime}$ we then have the push-forward functor $f_{*}: D(Y, k) \rightarrow D\left(Y^{\prime}, k\right)$ and the pull-back functor $f^{*}: D\left(Y^{\prime}, k\right) \rightarrow D(Y, k)$ (see $[$ Spa88]).

Definition 2.1. The equivariant derived category of sheaves on $X$ with coefficients in $k$ is the full subcategory $D_{G}(X, k)$ of $D\left(X_{G}, k\right)$ that contains all sheaves $\mathcal{F}$ for which there is a sheaf $\mathcal{F}_{X} \in D(X, k)$ such that $q^{*} \mathcal{F} \cong p^{*} \mathcal{F}_{X}$.

We denote by $D_{G}^{b}(X, k) \subset D_{G}(X, k)$ the full subcategory of objects with bounded cohomology, i.e. of objects that are contained in $D^{b}\left(X_{G}, k\right)$.

It turns out that the categories $D_{G}(X, k)$ and $D_{G}^{b}(X, k)$ are independent of the choice of $E G$. Since $p$ is a trivial fibration with contractible fibre $E G$, the functor $p^{*}: D(X, k) \rightarrow D(X \times E G, k)$ is a full embedding. We deduce that for $\mathcal{F} \in D_{G}(X, k)$ the sheaf $\mathcal{F}_{X} \in D(X, k)$ appearing in the definition above is unique up to unique isomorphism, so the map $\mathcal{F} \mapsto \mathcal{F}_{X}$ even extends to a functor For: $D_{G}(X, k) \rightarrow D(X, k)$.

2.2. The equivariant functor formalism. In order to ensure that all the functors that we introduce in the following exist we assume that $X$ is a complex algebraic variety endowed with its metric topology, and that $G$ is a Lie group acting continuously on $X$.

If $f: X \rightarrow Y$ is a continuous $G$-equivariant map then we get an induced map $f_{G}:=f \times_{G}$ id: $X_{G} \rightarrow Y_{G}$ and corresponding functors $f_{G}^{*}, f_{G *}, f_{G}^{!}$and $f_{G !}$ between the categories $D^{b}\left(X_{G}, k\right)$ and $D^{b}\left(Y_{G}, k\right)$. (Some care is needed in the definition of $f_{G}^{!}$and $f_{G \text { ! because }} X_{G}$ and $Y_{G}$ are not locally compact in general. In [BL94] this problem is overcome 
by considering $X_{G}$ as a direct limit of locally compact spaces. It is also possible to prove the existence and basic properties of $f_{G}^{!}$in a relative setting, see [SHS69].) It turns out that all four functors induce functors between the subcategories $D_{G}^{b}(X, k)$ and $D_{G}^{b}(Y, k)$. By abuse of notation we denote these functors by the symbols $f^{*}, f_{*}, f^{!}$and $f_{!}$.

For a $G$-stable subvariety $i: Y \hookrightarrow X$ and a sheaf $\mathcal{F} \in D_{G}^{b}(X, k)$ we define

$$
\mathcal{F}_{Y}:=i^{*} \mathcal{F}
$$

So $\mathcal{F}_{Y}$ is an object in $D_{G}^{b}(Y, k)$.

2.3. Equivariant cohomology. The equivariant cohomology $H_{G}^{\bullet}(X, k)$ of $X$ with coefficients in $k$ is the (ordinary) cohomology of the space $X_{G}$, i.e.

$$
H_{G}^{\bullet}(X, k):=H^{\bullet}\left(X_{G}, k\right) .
$$

In particular, the equivariant cohomology of a point is the cohomology of the classifying space

$$
B G:=p t_{G}=E G \times_{G} p t=E G / G
$$

of $G$.

Now let $\mathcal{F} \in D_{G}^{b}(X, k)$. The equivariant cohomology $\mathbb{H}_{G}^{\bullet}(\mathcal{F})$ of $X$ with coefficients in $\mathcal{F}$ is defined as follows. We denote by $\pi: X \rightarrow p t$ the map to a point. Then we have the object $\pi_{*} \mathcal{F} \in D_{G}^{b}(p t, k) \subset D^{b}(B G, k)$ and we define

$$
\mathbb{H}_{G}^{\bullet}(\mathcal{F}):=H^{\bullet}\left(\pi_{*} \mathcal{F}\right)
$$

where on the right we have the ordinary cohomology of $B G$ with coefficients in the sheaf $\pi_{*} \mathcal{F}$. This is naturally a graded module over $H_{G}^{\bullet}(p t, k)=H^{\bullet}(B G, k)$, so equivariant cohomology is a functor

$$
\mathbb{H}_{G}^{\bullet}: D_{G}^{b}(X, k) \rightarrow H_{G}^{\bullet}(p t, k)-\bmod ^{\mathbb{Z}} .
$$

Here and in the following we denote by $A$-mod $\mathbb{Z}^{\mathbb{Z}}$ the category of $\mathbb{Z}$ graded modules over a $\mathbb{Z}$-graded $\operatorname{ring} A$. For a graded $A$-module $M=$ $\bigoplus_{n \in \mathbb{Z}} M_{n}$ and $l \in \mathbb{Z}$ we denote by $M[l]$ the graded module obtained by a shift such that $M[l]_{n}=M_{l+n}$ for all $n \in \mathbb{Z}$.

Let $i: Y \hookrightarrow X$ be a locally closed $G$-stable subvariety and $\mathcal{F} \in D_{G}^{b}(X, k)$. The adjunction morphism id $\rightarrow i_{*} i^{*}$ yields a morphism $\mathcal{F} \rightarrow i_{*} i^{*} \mathcal{F}=$ $i_{*} \mathcal{F}_{Y}$. After applying equivariant cohomology this yields a homomorphism

$$
\mathbb{H}_{G}^{\bullet}(\mathcal{F}) \rightarrow \mathbb{H}_{G}^{\bullet}\left(i_{*} \mathcal{F}_{Y}\right)=\mathbb{H}_{G}^{\bullet}\left(\mathcal{F}_{Y}\right)
$$

of $H_{G}^{\bullet}(p t, k)$-modules. We call such a homomorphism a restriction homomorphism. 
2.4. The Mayer-Vietoris sequence. We will often make use of the equivariant Mayer-Vietoris sequence. Note that the equivariant statement is a straightforward consequence of the non-equivariant one (see, for example, [KS94, 2.6.28]).

Proposition 2.2. Let $X=U \cup V$ where $U, V \subset X$ are open and $G$ stable. Then, given any $\mathcal{F} \in D_{G}^{b}(X, k)$, we have a long exact sequence of equivariant cohomology

$$
\begin{aligned}
\cdots \rightarrow \mathbb{H}_{G}^{j-1}\left(\mathcal{F}_{U \cap V}\right) \rightarrow \mathbb{H}_{G}^{j}(\mathcal{F}) \rightarrow & \mathbb{H}_{G}^{j}\left(\mathcal{F}_{U}\right) \oplus \mathbb{H}_{G}^{j}\left(\mathcal{F}_{V}\right) \rightarrow \\
& \rightarrow \mathbb{H}_{G}^{j}\left(\mathcal{F}_{U \cap V}\right) \rightarrow \mathbb{H}_{G}^{j+1}(\mathcal{F}) \rightarrow \ldots
\end{aligned}
$$

2.5. The case of a torus. Let us suppose now that $G=T$ is a complex torus, i.e. a topological group isomorphic to $\left(\mathbb{C}^{\times}\right)^{r}$ for some $r>0$, endowed with the metric topology.

For $n \geq 0$ we consider the space $\left(\mathbb{C}^{n} \backslash\{0\}\right)^{r}$ together with the $T$ action given by

$$
\left(t_{1}, \ldots, t_{r}\right) \cdot\left(x_{1}, \ldots, x_{r}\right)=\left(t_{1} \cdot x_{1}, \ldots, t_{r} \cdot x_{r}\right) .
$$

The embeddings $\mathbb{C}^{n} \backslash\{0\} \rightarrow \mathbb{C}^{n+1} \backslash\{0\}$ that map $\left(z_{1}, \ldots, z_{n}\right)$ to $\left(z_{1}, \ldots, z_{n}, 0\right)$ define a direct system

$$
\cdots \rightarrow\left(\mathbb{C}^{n} \backslash\{0\}\right)^{r} \rightarrow\left(\mathbb{C}^{n+1} \backslash\{0\}\right)^{r} \rightarrow \ldots
$$

of $T$-spaces. The direct limit $\left(\mathbb{C}^{\infty} \backslash\{0\}\right)^{r}:=\lim \left(\mathbb{C}^{n} \backslash\{0\}\right)^{r}$ is a contractible space together with a topologically free $T$-action, hence can be chosen as a model for $E T$.

We denote by $X^{*}(T)$ the character lattice $\operatorname{Hom}\left(T, \mathbb{C}^{\times}\right)$of $T$. Let

$$
S_{k}:=S\left(X^{*}(T) \otimes_{\mathbb{Z}} k\right)
$$

be the symmetric algebra over the free $k$-module $X^{*}(T) \otimes_{\mathbb{Z}} k$, graded in such a way that $X^{*}(T) \otimes_{\mathbb{Z}} k \subset S_{k}$ is the homogeneous component of degree 2. Then the Borel homomorphism (cf. [Bri98], [Jan09]) gives a canonical identification

$$
S_{k} \stackrel{\sim}{\rightarrow} H^{\bullet}(B T, k)=H_{T}^{\bullet}(p t, k) .
$$

2.6. An attractive proposition. Now let $X$ be a complex $T$-variety. Recall that a $T$-fixed point $x \in X$ is called attractive if all weights of $T$ on the tangent space to $X$ at $x$ lie in an open half space of $X^{*}(T) \otimes_{\mathbb{Z}} \mathbb{R}$. If this is the case then one can find a one parameter subgroup $\alpha: \mathbb{C}^{\times} \rightarrow T$ and an open neighbourhood $U$ of $x$ such that

$$
\lim _{z \rightarrow 0} \alpha(z) \cdot y=x \text { for all } y \in U \text {. }
$$


If, in addition, $X$ is connected and affine, then $x$ is the unique $T$-fixed point of $X$ and (1) holds for all $y \in X$. In particular, the smallest $T$-stable open neighbourhood of $x \in X$ is $X$ itself.

Suppose for the remainder of this section that $X$ is connected and affine, and that $x \in X$ is an attractive fixed point. We denote by $i:\{x\} \rightarrow X$ the inclusion and by $\pi: X \rightarrow\{x\}$ the projection. If we apply the functor $\pi_{*}$ to the natural transformation id $\rightarrow i_{*} i^{*}$ we get a natural transformation $\pi_{*} \rightarrow \pi_{*} i_{*} i^{*}$. Since $\pi \circ i$ is the identity, we get a natural morphism

$$
\pi_{*} \rightarrow i^{*}
$$

of functors from $D_{T}^{b}(X, k)$ to $D_{T}^{b}(\{x\}, k)$.

The goal of the rest of this section is to prove the following (for similar statements in the non-equivariant or "weakly equivariant" setting see [Spr84] and [Bra03]):

Proposition 2.3. Suppose that $X$ is connected and affine and that $x \in X$ is an attractive fixed point. Then the morphism of functors $\pi_{*} \rightarrow i^{*}$ is an isomorphism.

We begin with some lemmata. Suppose we have a pair of Cartesian squares

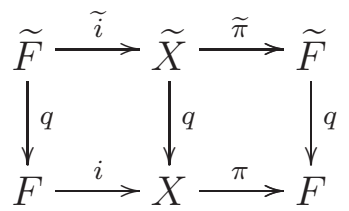

such that $q$ is smooth and surjective, and $\pi \circ i=\mathrm{id}$ (and hence $\tilde{\pi} \circ \tilde{i}=\mathrm{id}$ ). The adjunctions $\left(\pi^{*}, \pi_{*}\right)$ and $\left(\tilde{\pi}^{*}, \widetilde{\pi}_{*}\right)$ give morphisms of functors

$$
\pi_{*} \rightarrow i^{*} \text { and } \quad \tilde{\pi}_{*} \rightarrow \widetilde{i}^{*} .
$$

Lemma 2.4. Let $\mathcal{F} \in D^{b}(X, k)$. Then $\pi_{*} \mathcal{F} \rightarrow i^{*} \mathcal{F}$ is an isomorphism if and only if $\widetilde{\pi}_{*} q^{*} \mathcal{F} \rightarrow \widetilde{i}^{*} q^{*} \mathcal{F}$ is an isomorphism.

Proof. Because $q$ is surjective, $\pi_{*} \mathcal{F} \rightarrow i^{*} \mathcal{F}$ is an isomorphism if and only if $q^{*} \pi_{*} \mathcal{F} \rightarrow q^{*} i^{*} \mathcal{F}$ is an isomorphism. Now $q^{*} i^{*} \mathcal{F} \stackrel{\sim}{\rightarrow} \tilde{i}^{*} q^{*} \mathcal{F}$ and $q^{*} \pi_{*} \mathcal{F} \stackrel{\sim}{\rightarrow} \widetilde{\pi}_{*} q^{*} \mathcal{F}$ by smooth base change. Via these canonical isomorphisms we obtain a map

$$
\tilde{\pi}_{*} q^{*} \mathcal{F} \rightarrow \widetilde{i}^{*} q^{*} \mathcal{F}
$$

This is the same map (up to isomorphism) as that coming from the morphism $\widetilde{\pi}_{*} \rightarrow \widetilde{i}^{*}$ (cf. [BL94, Theorem 1.8].) 
Now suppose a torus $T$ contracts a variety $X$ onto a fixed locus $F \subset X$. Consider the diagram

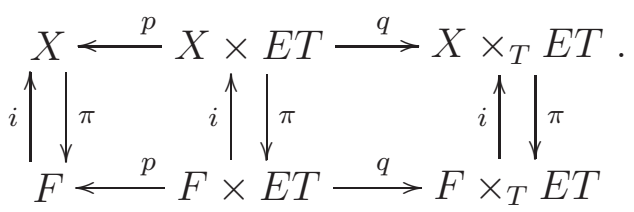

Both $p$ and $q$ are smooth, and so applying the above lemma twice we see that, given $\mathcal{F} \in D_{T}^{b}(X, k)$, we have that $\pi_{*} \mathcal{F} \rightarrow i^{*} \mathcal{F}$ is an isomorphism in $D_{T}^{b}(F, k)$ if and only if $\pi_{*} \operatorname{For}(\mathcal{F}) \rightarrow i^{*} \operatorname{For}(\mathcal{F})$ is.

Given a $G$-space $X$, let us call $\mathcal{F} \in D^{b}(X, k)$ naively equivariant if we have an isomorphism $m^{*} \mathcal{F} \rightarrow p^{*} \mathcal{F}$ where $m$ and $p$ denote the action and projection maps

$$
G \times X \underset{p}{\stackrel{m}{\longrightarrow}} X
$$

Note that, if $G$ acts freely on $X$ then pullback along $X \rightarrow X / G$ allows us to view any $\mathcal{F} \in D^{b}(X / G, k)$ as a naively equivariant sheaf on $X$. Note also that if $\mathcal{F}$ is naively equivariant for a group $G$, then it is also naively equivariant for any subgroup $H \subset G$.

Lemma 2.5. Suppose that $\mathcal{F} \in D_{G}^{b}(X, k)$. Then $\operatorname{For}(\mathcal{F})$ is naively equivariant for $G$.

Proof. Consider the quotient map $q: X \times E G \rightarrow X \times_{G} E G$. Then $q^{*} \mathcal{F}$ is naively equivariant for $G$. Then smooth base change applied to the projection $p: X \times E G \rightarrow X$ yields that $\operatorname{For}(\mathcal{F})=p_{*} q^{*} \mathcal{F}$ is naively equivariant for $G$.

We can now prove the attractive proposition:

Proof of Proposition 2.3. The above arguments reduce the proof of the above to showing that, if $\mathcal{F} \in D^{b}(X, k)$ is naively equivariant for the action of a one dimensional torus which contracts $X$ onto $x \in X$, then $\pi_{*} \mathcal{F} \rightarrow i^{*} \mathcal{F}$ is an isomorphism. But this is shown in [Spr82] (see also [Bra03] for another account of this argument).

\section{THE LOCALISATION HOMOMORPHISM}

Throughout this section we assume that $k$ is a unique factorisation domain and that $X$ is a normal complex algebraic variety (endowed with its metric topology), acted upon algebraically by a complex torus $T \cong\left(\mathbb{C}^{\times}\right)^{r}$. In addition, we assume the following: 
(A1) The torus acts on $X$ with only finitely many zero- and onedimensional orbits and the closure of each one-dimensional orbit is smooth.

(A2) $X$ admits a covering by open affine connected $T$-stable subvarieties, each of which contains an attractive (hence unique) fixed point.

Note that, by a result of Sumihiro (see [Sum74, KKLV89]), $X$ has a covering by open affine $T$-stable subvarieties, hence (A2) is automatically satisfied if $X$ is proper and each $T$-fixed point is attractive.

Let $X^{T} \subset X$ be the subspace of $T$-fixed points and $\mathcal{F} \in D_{T}^{b}(X, k)$. The restriction homomorphism associated to the inclusion $X^{T} \hookrightarrow X$,

$$
\mathbb{H}_{T}^{\bullet}(\mathcal{F}) \rightarrow \mathbb{H}_{T}^{\bullet}\left(\mathcal{F}_{X^{T}}\right)
$$

is called the localisation homomorphism.

As $X^{T}$ is a finite set we have $\mathbb{H}_{T}^{\bullet}\left(\mathcal{F}_{X^{T}}\right)=\bigoplus_{x \in X^{T}} \mathbb{H}_{T}^{\bullet}\left(\mathcal{F}_{x}\right)$. Following the results of [CS74] and [GKM98] we will show that for certain choices of $X, k$ and $\mathcal{F}$ the localisation map is injective and give an explicit description of its image. This is conveniently phrased in terms of moment graphs (cf. [BM01]), as it turns out that this image is determined by the restriction of $\mathcal{F}$ to the one-dimensional $T$-orbits in $X$.

3.1. One-dimensional orbits. Suppose that $E \subset X$ is a one-dimensional $T$-orbit. Then $E \cong T / \operatorname{Stab}_{T}(x)$ for any $x \in E$. Now $\operatorname{Stab}_{T}(x)$ is the kernel of a character $\alpha_{E} \in X^{*}(T)$ which is well-defined up to a sign. From now on we fix a choice of $\alpha_{E}$ for each one-dimensional orbit $E$ in $X$. Nothing that follows depends on this choice.

As before we denote by $S_{k}$ the $\mathbb{Z}$-graded symmetric algebra of the free $k$-module $X^{*}(T) \otimes_{\mathbb{Z}} k$ and identify it with the $T$-equivariant cohomology of a point with coefficients in $k$. Given $\alpha \in X^{*}(T)$ we often abuse notation and denote by $\alpha$ as well the image of $\alpha \otimes 1 \in X^{*}(T) \otimes_{\mathbb{Z}} k$ in $S_{k}$.

Now $\alpha_{E}$ acts as zero on $H_{T}^{\bullet}(E, k)$ (see, for example, [Jan03, Section 1.9]). As $\mathbb{H}_{\mathrm{T}}^{\bullet}\left(\mathcal{F}_{E}\right)$ is a $H_{T}^{\bullet}(E, k)$-module, we conclude:

Lemma 3.1. For any one-dimensional $T$-orbit $E$ in $X$ and any $\mathcal{F} \in$ $D_{T}^{b}(X, k)$ we have $\alpha_{E} \mathbb{H}_{\mathrm{T}}^{\bullet}\left(\mathcal{F}_{E}\right)=0$.

3.2. The localisation theorem - part I. For any closed connected subgroup $\Gamma$ of $T$ we let $X^{\Gamma}$ be the subset of $\Gamma$-fixed points in $X$. Let us fix a closed subspace $Z \subset X$ which is a discrete union

$$
Z=X^{\Gamma_{1}} \sqcup \cdots \sqcup X^{\Gamma_{n}}
$$


of the fixed points in $X$ of finitely many connected subtori $\Gamma_{1}, \ldots, \Gamma_{n} \subset$ $T$. We set

$$
P^{Z}:=\left\{\begin{array}{l|l}
\alpha_{E} \in X^{*}(T) & \begin{array}{c}
E \text { is a one-dimensional } \\
T \text {-orbit in } X \backslash Z
\end{array}
\end{array}\right\}
$$

and define

$$
s^{Z}:=\prod_{\alpha \in P^{Z}} \alpha \in S_{k}
$$

In addition to (A1) and (A2) we assume from now on:

(A3) for each one-dimensional orbit $E$ in $X$ the image of $\alpha_{E} \in X^{*}(T)$ is non-zero in $S_{k}$.

(Of course this condition is vacuous if the characteristic of $k$ is 0 .)

We now come to the first part of the localisation theorem. In the characteristic 0 case it is due to Goresky, Kottwitz and MacPherson (cf. [GKM98]).

Theorem 3.2. Assume that the assumptions (A1), (A2) and (A3) hold and let $\mathcal{F} \in D_{T}^{b}(X, k)$. Suppose that $\mathbb{H}_{T}^{\bullet}(\mathcal{F})$ is a graded free $S_{k}$-module. Then the restriction homomorphism

$$
\mathbb{H}_{T}^{*}(\mathcal{F}) \rightarrow \mathbb{H}_{T}^{*}\left(\mathcal{F}_{Z}\right)
$$

is injective and becomes an isomorphism after inverting $s^{Z} \in S_{k}$, i.e. after applying the functor $\cdot \otimes_{S_{k}} S_{k}\left[1 / s^{Z}\right]$.

The proof of the theorem will take up the rest of this section. We follow Brion's account [Bri98, Section 2] of the characteristic 0 case quite closely, but at points some additional care is needed.

Let $K \cong\left(S^{1}\right)^{r} \subset T \cong\left(\mathbb{C}^{\times}\right)^{r}$ be the maximal compact subtorus of $T$. We can regard $X$ as a $K$-space via restriction of the action. This yields a functor

$$
\operatorname{res}_{K}^{T}: D_{T}^{b}(X, k) \rightarrow D_{K}^{b}(X, k) .
$$

As $T / K$ is contractible, for any equivariant sheaf $\mathcal{G} \in D_{T}^{b}(X, k)$ restriction gives an isomorphism

$$
\mathbb{H}_{T}^{\bullet}(\mathcal{G}) \stackrel{\sim}{\rightarrow} \mathbb{H}_{K}^{\bullet}\left(\operatorname{res}_{K}^{T} \mathcal{G}\right) .
$$

In particular, we have a canonical isomorphism $H_{K}^{\bullet}(p t, k) \cong S_{k}$. In the following we write $\mathbb{H}_{K}^{\bullet}(\mathcal{G})$ for $\mathbb{H}_{K}^{\bullet}\left(\operatorname{res}_{K}^{T} \mathcal{G}\right)$. Hence, for the proof of Theorem 3.2, it is enough to consider the restriction homomorphism

$$
\mathbb{H}_{K}^{\bullet}(\mathcal{F}) \rightarrow \mathbb{H}_{K}^{\bullet}\left(\mathcal{F}_{Z}\right)
$$

and to show that it is injective and becomes an isomorphism after inverting $s^{Z}$. 
Before we prove this we need a couple of preliminary results. We state them for the $K$-equivariant cohomology, however all lemmata except Lemma 3.5 are true with $T$ in place of $K$.

First we assume that $X=V$ is a finite dimensional $T$-module. Let $P \subset X^{*}(T)$ be the characters occurring in $V$ and $s=\prod_{\chi \in P} \chi \in S_{k}$ their product. Here is the first step towards the localization theorem.

Lemma 3.3. If $\mathcal{F} \in D_{K}^{b}(V \backslash\{0\}, k)$ then $\mathbb{H}_{K}^{\bullet}(\mathcal{F})$ is annihilated by a power of $s$.

Proof. Fix an isomorphism

$$
V \cong \mathbb{C}_{\chi_{1}} \oplus \mathbb{C}_{\chi_{2}} \oplus \cdots \oplus \mathbb{C}_{\chi_{m}}
$$

where $\chi_{1}, \chi_{2}, \ldots, \chi_{m} \in P$. (Here, given $\chi \in X^{*}(T), \mathbb{C}_{\chi}$ denotes the onedimensional $T$-module with character $\chi$.) We will use this isomorphism to write elements of $V$ as $\left(x_{j}\right)_{1 \leq j \leq m}$. For any $1 \leq i \leq m$ consider the subset

$$
U_{i}=\left\{\left(x_{j}\right) \in V \mid x_{i} \neq 0\right\} .
$$

Projection gives us an equivariant map $U_{i} \rightarrow \mathbb{C}_{\chi_{i}}^{\times}$. By Lemma 3.1, the equivariant cohomology $\mathbb{H}_{K}^{\bullet}(\mathcal{G})$ of each $\mathcal{G} \in D_{K}^{b}\left(U_{i}, k\right)$ is annihilated by $\chi_{i}$.

However, $V \backslash\{0\}$ is covered by the sets $U_{i}$ for $1 \leq i \leq m$ and the Mayer-Vietoris sequence allows us to conclude that $\mathbb{H}_{K}^{\bullet}(\mathcal{F})$ is annihilated by a power of $s$.

Now let $Z \subset X$ be as before. From the above we deduce the second step:

Lemma 3.4. If $\mathcal{F} \in D_{K}^{b}(X \backslash Z, k)$ then $\mathbb{H}_{K}^{\bullet}(\mathcal{F})$ is annihilated by a power of $s^{Z}$.

Proof. First we assume that $X$ is affine and connected and contains an attractive fixed point. In this case $Z$ is necessarily of the form $X^{\Gamma}$ for a closed subtorus $\Gamma \subset T$. We recall an argument due to Brion (cf. [Ara98, Proposition 3.2.1-1], or the proof of Theorem 17 in [Bri98]) which constructs a finite $T$-equivariant map

$$
\pi: X \rightarrow V
$$

where $V$ is a $T$-module with weights corresponding bijectively to the one-dimensional orbits of $T$ in $X$. Brion's construction is as follows:

For each one-dimensional orbit $E \subset X, \bar{E}$ is smooth and hence isomorphic, as a $T$-space, to $\mathbb{C}_{\alpha_{E}}$. For each such orbit we may find a regular function $\pi_{E}: X \rightarrow \mathbb{C}_{\alpha_{E}}$ such that the restriction of $\pi_{E}$ to $\bar{E}$ 
is an equivariant isomorphism of affine spaces. Taking the direct sum over all such $\pi_{E}$ yields a map

$$
X \stackrel{\pi}{\rightarrow} V:=\bigoplus_{E} \mathbb{C}_{\alpha_{E}}
$$

We claim that $\pi$ is finite. Because $x \in X$ is attractive, we can find a rank one subtorus of $T$ inducing a positive grading on the regular functions on $X$. By the graded Nakayama lemma $\pi$ is finite if and only if $\pi^{-1}(0)$ is finite. If $\pi^{-1}(0)$ is not finite, then it contains a one-dimensional $T$-orbit (again by the attractiveness of $x$ ), but this contradicts the construction.

Now let $V^{\Gamma} \subset V$ be the subspace of $\Gamma$-fixed points. Because each fibre of $\pi$ is finite and $\pi$ is equivariant it follows that $\pi^{-1}\left(V^{\Gamma}\right)=X^{\Gamma}$. Choose a decomposition

$$
V=V^{\prime} \oplus V^{\Gamma}
$$

of $T$-modules and let $V \rightarrow V^{\prime}$ denote the projection. We get an induced map

$$
\pi^{\prime}: X \backslash X^{\Gamma} \rightarrow V^{\prime} \backslash\{0\}
$$

and the result follows from Lemma 3.3 because

$$
\mathbb{H}_{K}^{\bullet}(\mathcal{F}) \cong \mathbb{H}_{K}^{\bullet}\left(\pi_{*}^{\prime} \mathcal{F}\right) .
$$

Hence we proved the lemma in the case of affine $X$.

By our assumption (A2), the general case follows from the MayerVietoris sequence.

Lemma 3.5. For any equivariant sheaf $\mathcal{F} \in D_{K}^{b}(X, k)$ we have an isomorphism

$$
\mathbb{H}_{K}^{\bullet}\left(\mathcal{F}_{Z}\right) \cong \lim _{\rightarrow} \mathbb{H}_{K}^{\bullet}\left(\mathcal{F}_{U}\right)
$$

where the direct limit takes place over all $K$-stable open neighbourhoods $U$ of $Z$.

Proof. By assumption $X$ has a covering by open subvarieties, all isomorphic to closed subvarieties of affine spaces with linear $T$-actions. Thus we may choose a basis of open neighbourhoods $\left\{U_{i}\right\}_{i \in I}$ of $Z$ which are $K$-stable. (This is where we need the compactness of $K$.)

Now we may write $E K$ as a countable direct limit of (finite dimensional) manifolds with free $K$-action (for example, by taking $E K=E T$ as in Section 2.5). Hence $X_{K}$ can be written as a countable union of compact subsets. Because $X_{K}$ is regular, we conclude that $X_{K}$ is paracompact (cf. [MS74, Section 5.8] and [Dug66, Theorem 6.5]). It is 
straightforward to see that $\left\{\left(U_{i}\right)_{K}\right\}_{i \in I}$ give a basis of open neighbourhoods of $Z_{K}$. It then follows from [KS94, Remark 2.6.9] that we have an isomorphism

$$
\mathbb{H}_{K}^{\bullet}\left(\mathcal{F}_{Z}\right)=\mathbb{H}^{\bullet}\left(\mathcal{F}_{Z_{K}}\right) \cong \lim _{\rightarrow} \mathbb{H}^{\bullet}\left(\mathcal{F}_{\left(U_{i}\right)_{K}}\right)=\lim _{\rightarrow} \mathbb{H}_{K}^{\bullet}\left(\mathcal{F}_{U}\right)
$$

as claimed.

Now we are ready to prove Theorem 3.2.

Proof. Let $\mathcal{F} \in D_{T}^{b}(X, k)$ and assume that $\mathbb{H}_{T}^{\bullet}(\mathcal{F})$ is free as an $S_{k^{-}}$ module. We have to show that the restriction map

$$
\mathbb{H}_{K}^{\bullet}(\mathcal{F}) \rightarrow \mathbb{H}_{K}^{\bullet}\left(\mathcal{F}_{Z}\right)
$$

is injective, and becomes an isomorphism after inverting $s^{Z}$.

Let $U$ be an open $K$-stable neighbourhood of $Z \subset X$. We have inclusions

$$
U \stackrel{j}{\hookrightarrow} X \stackrel{i}{\hookrightarrow} X \backslash U
$$

and hence a distinguished triangle:

$$
i_{i} i^{!} \mathcal{F} \rightarrow \mathcal{F} \rightarrow j_{*} j^{*} \mathcal{F} \stackrel{[1]}{\rightarrow} \text {. }
$$

Applying Lemma 3.4 (and remembering that $i_{*} \cong i_{\text {! }}$ ) we deduce that $\mathbb{H}_{K}^{\bullet}\left(i_{!} i ! \mathcal{F}\right)$ is annihilated by a power of $s^{Z}$. As $\mathbb{H}_{K}^{\bullet}(\mathcal{F})$ is free, the restriction map $\mathbb{H}_{K}^{\bullet}(\mathcal{F}) \rightarrow \mathbb{H}_{K}^{\bullet}\left(\mathcal{F}_{U}\right)$ is injective. It also follows that it becomes an isomorphism after inverting $s^{Z}$.

To finish the proof, note that, by Lemma 3.5,

$$
\mathbb{H}_{K}^{\bullet}\left(\mathcal{F}_{Z}\right) \cong \lim _{\rightarrow} \mathbb{H}_{K}^{\bullet}\left(\mathcal{F}_{U}\right)
$$

Because $\mathbb{H}_{K}^{\bullet}(\mathcal{F}) \rightarrow \mathbb{H}_{K}^{\bullet}\left(\mathcal{F}_{U}\right)$ is injective for all $U$ it follows that $\mathbb{H}_{K}^{\bullet}(\mathcal{F}) \rightarrow$ $\mathbb{H}_{K}^{\bullet}\left(\mathcal{F}_{Z}\right)$ is injective. Lastly, this map becomes an isomorphism after inverting $s^{Z}$ because the direct limit commutes with tensor products.

\section{The IMAge of The LOCALISATION HOMOMORPHISM}

We are now going to describe the image of the localisation homomorphism under a certain further restriction on the ring $k$ which is called the GKM-condition. For this it is convenient to use the language of sheaves on moment graphs. We start by recalling the main definitions and constructions in the theory of moment graphs. In particular, we define the $\mathbb{Z}$-graded category $\mathcal{G}$ - $\bmod _{k}^{\mathbb{Z}}$ of $k$-sheaves on a moment graph $\mathcal{G}$ and associate to any such sheaf $\mathscr{F}$ its space of global sections $\Gamma(\mathscr{F})$.

To a $T$-space $X$ with finitely many zero- and one-dimensional orbits we associate a moment graph $\mathcal{G}_{X}$ and define a functor

$$
\mathbb{W}: D_{T}^{b}(X, k) \rightarrow \mathcal{G}_{X}-\bmod _{k}^{\mathbb{Z}}
$$


between $\mathbb{Z}$-graded categories. We then show that under some assumptions on $\mathcal{F} \in D_{T}^{b}(X, k)$, the equivariant cohomology of $X$ with coefficients in $\mathcal{F}$ coincides with the space of global sections of $\mathbb{W}(\mathcal{F})$, i.e.

$$
\mathbb{H}_{\mathrm{T}}^{\bullet}(\mathcal{F})=\Gamma(\mathbb{W}(\mathcal{F})) \text {. }
$$

4.1. Sheaves on moment graphs. Let $Y \cong \mathbb{Z}^{r}$ be a lattice of finite rank.

Definition 4.1. An (unordered) moment graph $\mathcal{G}$ over $Y$ is given by the following data:

- $A \operatorname{graph}(\mathcal{V}, \mathcal{E})$ with set of vertices $\mathcal{V}$ and set of edges $\mathcal{E}$.

- $A \operatorname{map} \alpha: \mathcal{E} \rightarrow Y \backslash\{0\}$.

We assume that two vertices of a moment graph are connected by at most one edge.

Let $\mathcal{G}=(\mathcal{V}, \mathcal{E}, \alpha)$ be a moment graph. We write $E: x-y$ for an edge $E$ that connects the vertices $x$ and $y$. If we also want to denote the label $\alpha=\alpha(E)$ of $E$, then we write $E: x \stackrel{\alpha}{-} y$. As before we denote by $S_{k}=S\left(Y \otimes_{\mathbb{Z}} k\right)$ the symmetric algebra of $Y$ over $k$, which we consider as a graded algebra with $Y \otimes_{\mathbb{Z}} k$ sitting in degree 2 .

Definition 4.2. A $k$-sheaf $\mathscr{M}$ on a moment graph $\mathcal{G}$ is given by the following data:

- a graded $S_{k}$-module $\mathscr{M}^{x}$ for any vertex $x \in \mathcal{V}$,

- a graded $S_{k}$-module $\mathscr{M}^{E}$ with $\alpha(E) \mathscr{M}^{E}=0$ for any $E \in \mathcal{E}$,

- a homomorphism $\rho_{x, E}: \mathscr{M}^{x} \rightarrow \mathscr{M}^{E}$ of graded $S_{k}$-modules for any vertex $x$ lying on the edge $E$.

For a $k$-sheaf $\mathscr{M}$ on $\mathcal{G}$ and $l \in \mathbb{Z}$ we denote by $\mathscr{M}[l]$ the shifted $k$-sheaf with stalks $\mathscr{M}[l]^{x}=\left(\mathscr{M}^{x}\right)[l], \mathscr{M}[l]^{E}=\left(\mathscr{M}^{E}\right)[l]$ and shifted $\rho$-homomorphisms. A morphism $f: \mathscr{M} \rightarrow \mathscr{N}$ between $k$-sheaves $\mathscr{M}$ and $\mathscr{N}$ on $\mathcal{G}$ is given by a collection of homomorphisms of graded $S_{k}$-modules $f^{x}: \mathscr{M}^{x} \rightarrow \mathscr{N}^{x}$ and $f^{E}: \mathscr{M}^{E} \rightarrow \mathscr{N}^{E}$ for all vertices $x$ and edges $E$ that are compatible with the maps $\rho$, i.e. such that the diagram

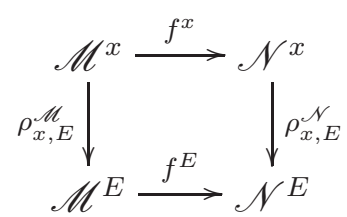

commutes for all vertices $x$ that lie on the edge $E$. We denote by $\mathcal{G}$-mod $\mathbb{Z}_{k}^{\mathbb{Z}}$ the category whose objects are $k$-sheaves on $\mathcal{G}$ and whose morphisms are the morphisms between $k$-sheaves. It is $\mathbb{Z}$-graded by the functor $\mathscr{M} \mapsto \mathscr{M}[1]$. 
4.2. Sections of sheaves and the structure algebra. The structure algebra over $k$ of a moment graph $\mathcal{G}$ is

$$
\mathcal{Z}_{k}=\left\{\left(z_{x}\right) \in \prod_{x \in \mathcal{V}} S_{k} \mid \begin{array}{c}
z_{x} \equiv z_{y} \bmod \alpha(E) \\
\text { for all edges } E: x-y
\end{array}\right\} .
$$

Coordinatewise addition and multiplication makes $\mathcal{Z}_{k}$ into an $S_{k}$-algebra. It is $\mathbb{Z}$-graded if we consider the product in the definition in the graded sense.

Let $\mathscr{M}$ by a $k$-sheaf on $\mathcal{G}$. For any subset $\mathcal{I}$ of $\mathcal{V}$ we define the space of sections of $\mathscr{M}$ over $\mathcal{I}$ by

$$
\Gamma(\mathcal{I}, \mathscr{M}):=\left\{\begin{array}{l|l}
\left(m_{x}\right) \in \prod_{x \in \mathcal{I}} \mathscr{M}^{x} & \begin{array}{c}
\rho_{x, E}\left(m_{x}\right)=\rho_{y, E}\left(m_{y}\right) \\
\text { for all edges } E: x- \\
\text { with } x, y \in \mathcal{I}
\end{array}
\end{array}\right\} .
$$

Coordinatewise multiplication makes $\Gamma(\mathcal{I}, \mathscr{M})$ into a $\mathcal{Z}_{k}$-module (as $\alpha(E) \rho_{x, E}\left(m_{x}\right)=0$ for any edge $E$ with vertex $\left.x\right)$. Again it is a graded module when the product is taken in the category of graded $S_{k}$-modules.

We call the space $\Gamma(\mathscr{M}):=\Gamma(\mathcal{V}, \mathscr{M})$ the space of global sections. If $\mathcal{I} \subset \mathcal{J}$ are two subsets of $\mathcal{V}$, then the canonical projection $\bigoplus_{x \in \mathcal{J}} \mathscr{M}^{x} \rightarrow$ $\bigoplus_{x \in \mathcal{I}} \mathscr{M}^{x}$ induces a restriction map $\Gamma(\mathcal{J}, \mathscr{M}) \rightarrow \Gamma(\mathcal{I}, \mathscr{M})$.

4.3. The costalks of a sheaf. Let $\mathscr{M}$ be a $k$-sheaf on $\mathcal{G}$ and let $x$ be a vertex. Then we define the costalk $\mathscr{M}_{x}$ of $\mathscr{M}$ at $x$ to be the $S_{k}$-module

$$
\mathscr{M}_{x}:=\left\{m \in \mathscr{M}^{x} \mid \rho_{x, E}(m)=0 \text { for all edges } E \text { that contain } x\right\} .
$$

We can identify $\mathscr{M}_{x}$ in an obvious way with the kernel of the restriction homomorphism $\Gamma(\mathcal{V}, \mathscr{M}) \rightarrow \Gamma(\mathcal{V} \backslash\{x\}, \mathscr{M})$.

4.4. The moment graph associated to a $T$-variety. To a complex $T$-variety $X$ satisfying (A1) we associate the following moment graph $\mathcal{G}_{X}=(\mathcal{V}, \mathcal{E}, \alpha)$ over the lattice $X^{*}(T)$ :

- We set $\mathcal{V}:=X^{T}$.

- The vertices $x$ and $y, x \neq y$, are connected by an edge if there is a one-dimensional orbit $E$ such that $\bar{E}=E \cup\{x, y\}$. We denote this edge by $E$ as well.

- We let $\alpha(E)=\alpha_{E} \in X^{*}(T)$ be the chosen character.

Note that only those one-dimensional orbits $E$ in $X$ give rise to an edge that pick up two distinct fixed points in their closure. 
4.5. The functor $\mathbb{W}$. Suppose that $E \subset X$ is a one-dimensional $T$ orbit, and suppose that $x \in \bar{E}$ is a fixed point in its closure. Let $\mathcal{F}$ be an object in $D_{T}^{b}(X, k)$. Then the restriction homomorphism

$$
\mathbb{H}_{\mathrm{T}}^{\bullet}\left(\mathcal{F}_{E \cup\{x\}}\right) \rightarrow \mathbb{H}_{\mathrm{T}}^{\bullet}\left(\mathcal{F}_{x}\right)
$$

is an isomorphism by the attractive Proposition 2.3. Hence we can define a homomorphism $\rho_{x, E}$ from $\mathbb{H}_{\mathrm{T}}^{\bullet}\left(\mathcal{F}_{x}\right)$ to $\mathbb{H}_{\mathrm{T}}^{\bullet}\left(\mathcal{F}_{E}\right)$ by composing the inverse of the above homomorphism with the restriction homomorphism $\mathbb{H}_{\mathrm{T}}^{\bullet}\left(\mathcal{F}_{E \cup\{x\}}\right) \rightarrow \mathbb{H}_{\mathrm{T}}^{\bullet}\left(\mathcal{F}_{E}\right):$

$$
\rho_{x, E}: \mathbb{H}_{\mathrm{T}}^{\bullet}\left(\mathcal{F}_{x}\right) \stackrel{\sim}{\leftarrow} \mathbb{H}_{\mathrm{T}}^{\bullet}\left(\mathcal{F}_{E \cup\{x\}}\right) \rightarrow \mathbb{H}_{\mathrm{T}}^{\bullet}\left(\mathcal{F}_{E}\right) .
$$

Now we can define the functor $\mathbb{W}$. To an equivariant sheaf $\mathcal{F} \in$ $D_{T}^{b}(X, k)$ on $X$ we associate the following $k$-sheaf $\mathbb{W}(\mathcal{F})$ on $\mathcal{G}_{X}$ :

- For a vertex $x \in \mathcal{V}$ we set $\mathbb{W}(\mathcal{F})^{x}:=\mathbb{H}_{\mathrm{T}}^{\bullet}\left(\mathcal{F}_{x}\right)$.

- For a one-dimensional orbit $E$ we set $\mathbb{W}(\mathcal{F})^{E}:=\mathbb{H}_{T}^{\bullet}\left(\mathcal{F}_{E}\right)$ (note that $\alpha_{E} \mathbb{H}_{\mathrm{T}}^{\bullet}\left(\mathcal{F}_{E}\right)=0$ by Lemma 3.1).

- In case that $x \in \bar{E}$ we let $\rho_{x, E}: \mathbb{W}(\mathcal{F})^{x} \rightarrow \mathbb{W}(\mathcal{F})^{E}$ be the map constructed above.

This construction clearly extends to a functor $\mathbb{W}: D_{T}^{b}(X, k) \rightarrow \mathcal{G}_{X}-\bmod _{k}^{\mathbb{Z}}$.

4.6. The case $X=\mathbb{P}^{1}$. Suppose that $T$ acts linearly on $\mathbb{P}^{1}$ via a non-trivial character $\alpha$. In this case the moment graph is

$$
0 \stackrel{\alpha}{-} \infty \text {. }
$$

For $\mathcal{F} \in D_{T}^{b}\left(\mathbb{P}^{1}, k\right)$ the sheaf $\mathbb{W}(\mathcal{F})$ consists of the stalks $\mathbb{H}_{\mathrm{T}}^{\bullet}\left(\mathcal{F}_{0}\right)$, $\mathbb{H}_{\mathrm{T}}^{\bullet}\left(\mathcal{F}_{\infty}\right)$ and the space $\mathbb{H}_{\mathrm{T}}^{\bullet}\left(\mathcal{F}_{\mathbb{C}^{\times}}\right)$together with the maps

$$
\mathbb{H}_{\mathrm{T}}^{\bullet}\left(\mathcal{F}_{0}\right) \stackrel{\rho_{0, \mathbb{C} \times}}{\longrightarrow} \mathbb{H}_{\mathrm{T}}^{\bullet}\left(\mathcal{F}_{\mathbb{C}^{\times}}\right) \stackrel{\rho_{\infty}, \mathbb{C}^{\times}}{\longleftarrow} \mathbb{H}_{\mathrm{T}}^{\bullet}\left(\mathcal{F}_{\infty}\right) .
$$

A consequence of the Mayer-Vietoris sequence is the following lemma.

Lemma 4.3. Let $\mathcal{F} \in D_{T}^{b}\left(\mathbb{P}^{1}, k\right)$. Then the image of the restriction homomorphism $\mathbb{H}_{\mathrm{T}}^{\bullet}(\mathcal{F}) \rightarrow \mathbb{H}_{\mathrm{T}}^{\bullet}\left(\mathcal{F}_{0}\right) \oplus \mathbb{H}_{\mathrm{T}}^{\bullet}\left(\mathcal{F}_{\infty}\right)$ is $\left\{\left(z_{0}, z_{\infty}\right) \mid \rho_{0, \mathbb{C} \times}\left(z_{0}\right)=\right.$ $\left.\rho_{\infty, \mathbb{C} \times}\left(z_{\infty}\right)\right\}$.

4.7. The localisation theorem - part II. Now we assume that $X$ satisfies the assumptions (A1), (A2) and (A3). Let $\mathcal{F} \in D_{T}^{b}(X, k)$. If $\mathbb{H}_{T}^{\bullet}(\mathcal{F})$ is a free $S_{k}$-module, then Theorem 3.2 shows that we can view $\mathbb{H}_{T}^{\bullet}(\mathcal{F})$ as a submodule of $\bigoplus_{x \in X^{T}} \mathbb{H}_{T}^{\bullet}\left(\mathcal{F}_{x}\right)=\bigoplus_{x \in X^{T}} \mathbb{W}(\mathcal{F})^{x}$. The space of global sections $\Gamma(\mathbb{W}(\mathcal{F})$ ) is a submodule of this direct sum as well. In this section we want to prove that these two submodules coincide.

We need some more notation. For $\alpha \in X^{*}(T)$ let us define $X^{\alpha}$ to be the subvariety of all $T$-fixed points in $X$ and all one-dimensional orbits 
$E \subset X$ such that $k \alpha \cap k \alpha_{E} \neq 0$. Then $X^{0}=X^{T}$ for all rings $k$, but in general $X^{\alpha}$ depends on the ring $k$. We define

$$
P^{\alpha}:=\left\{\begin{array}{l|l}
\alpha_{E} \in X^{*}(T) & \begin{array}{c}
E \text { is a one-dimensional } \\
T \text {-orbit in } X \backslash X^{\alpha}
\end{array}
\end{array}\right\}
$$

and

$$
s^{\alpha}:=\prod_{\alpha_{E} \in P^{\alpha}} \alpha_{E} \in S_{k}
$$

We need some additional assumptions on our data:

(A4a) For any $\alpha \in X^{*}(T)$ the space $X^{\alpha}$ is a disjoint union of points and $\mathbb{P}^{1}$ 's.

(A4b) If $E$ is a one-dimensional $T$-orbit and $n \in \mathbb{Z}$ is such that $\alpha_{E}$ is divisible by $n$ in $X^{*}(T)$, then $n$ is invertible in $k$.

Note that (A4a) and (A4b) imply that the greatest common divisor of $s^{\alpha}$ for all $\alpha \in X^{*}(T)$ is 1 . For the proof of the next theorem we will only need this fact, but we need the stronger statements (A4a) and (A4b) later. Note also that (A4a) guarantees that we can apply Theorem 3.2 with $Z=X^{\alpha}$ and $s^{Z}=s^{\alpha}$.

Let $\mathcal{G}_{X}$ be the moment graph associated to $X$. For $\alpha \in X^{*}(T)$ we denote by $\mathcal{G}_{X}^{\alpha}$ the moment graph obtained from $\mathcal{G}_{X}$ by deleting all edges $E$ with $k \alpha_{E} \cap k \alpha=0$. Then (A4a) is equivalent to:

$(\mathrm{A} 4 \mathrm{a})^{\prime}$ The moment graph $\mathcal{G}_{X}^{\alpha}$ is a (discrete) union of moment graphs with only one or two vertices.

Now we can state the second part of the localisation theorem.

Theorem 4.4. Suppose that (A1), (A2), (A3), (A4a) and (A4b) hold. Let $\mathcal{F} \in D_{T}^{b}(X, k)$ and suppose that $\mathbb{H}_{T}^{\bullet}(\mathcal{F})$ and $\mathbb{H}_{T}^{\bullet}\left(\mathcal{F}_{X^{T}}\right)$ are free $S_{k^{-}}$ modules. Then

$$
\mathbb{H}_{T}^{\bullet}(\mathcal{F})=\Gamma(\mathbb{W}(\mathcal{F}))
$$

as submodules of $\bigoplus_{x \in X^{T}} \mathbb{H}_{T}^{\bullet}\left(\mathcal{F}_{x}\right)=\bigoplus_{x \in X^{T}} \mathbb{W}(\mathcal{F})^{x}$.

For the proof of the above statement we use similar arguments as the ones given in [CS74], [GKM98] or [Bri98]. Again we follow [Bri98] closely.

Proof. As a first step let $\mathcal{F} \in D_{T}^{b}(X, k)$ be any sheaf and $\alpha \in X^{*}(T)$. Let $\Gamma^{\alpha}(\mathbb{W}(\mathcal{F}))$ be the sections of the sheaf $\mathbb{W}(\mathcal{F})$ on the moment graph $\mathcal{G}_{X}^{\alpha}$ (so we only consider the edges $E$ with $k \alpha_{E} \cap k \alpha \neq 0$ ). By (A3),

$$
\Gamma(\mathbb{W}(\mathcal{F}))=\bigcap_{\alpha \in X^{*}(T)} \Gamma^{\alpha}(\mathbb{W}(\mathcal{F}))
$$


By (A4a), $X^{\alpha}$ is a discrete union of points and $\mathbb{P}^{1}$ 's. Hence, if we denote by $r_{\alpha}: \mathbb{H}_{\mathrm{T}}^{\bullet}\left(\mathcal{F}_{X^{\alpha}}\right) \rightarrow \mathbb{H}_{\mathrm{T}}^{\bullet}\left(\mathcal{F}_{X^{T}}\right)$ the restriction map, then Lemma 4.3 yields $\Gamma^{\alpha}(\mathbb{W}(\mathcal{F}))=r_{\alpha}\left(\mathbb{H}_{\mathrm{T}}^{\bullet}\left(\mathcal{F}_{X^{\alpha}}\right)\right)$. Hence:

$$
\Gamma(\mathbb{W}(\mathcal{F}))=\bigcap_{\alpha \in X^{*}(T)} r_{\alpha}\left(\mathbb{H}_{\mathrm{T}}^{\bullet}\left(\mathcal{F}_{X^{\alpha}}\right)\right)
$$

So we have to show that $\mathbb{H}_{\mathrm{T}}^{\bullet}(\mathcal{F})=\bigcap_{\alpha \in X^{*}(T)} r_{\alpha}\left(\mathbb{H}_{\mathrm{T}}^{\bullet}\left(\mathcal{F}_{X^{\alpha}}\right)\right)$.

Clearly $\mathbb{H}_{\mathrm{T}}^{\bullet}(\mathcal{F})$ is contained in the intersection $\bigcap_{\alpha \in X^{*}(T)} r_{\alpha}\left(\mathbb{H}_{\mathrm{T}}^{\bullet}\left(\mathcal{F}_{X^{\alpha}}\right)\right)$. Hence it remains to show that if $f \in \mathbb{H}_{T}^{\bullet}\left(\mathcal{F}_{X^{T}}\right)$ is in $r_{\alpha}\left(\mathbb{H}_{\mathrm{T}}^{\bullet}\left(\mathcal{F}_{X^{\alpha}}\right)\right)$ for all $\alpha \in X^{*}(T)$, then $f$ is contained in $\mathbb{H}_{T}^{\bullet}(\mathcal{F})$.

By Theorem 3.2 the injection $i: \mathbb{H}_{\mathrm{T}}^{\bullet}(\mathcal{F}) \rightarrow \mathbb{H}_{\mathrm{T}}^{\bullet}\left(\mathcal{F}_{X^{T}}\right)$ becomes an isomorphism after inverting $s^{0}$. By assumption, $\mathbb{H}_{T}^{\bullet}(\mathcal{F})$ is a free $S_{k}$-module. We choose a basis $e_{1}, \ldots, e_{m}$ for $\mathbb{H}_{T}^{\bullet}(\mathcal{F})$ and denote by $e_{1}^{*}, \ldots, e_{m}^{*} \in \operatorname{Hom}\left(\mathbb{H}_{T}^{\bullet}(\mathcal{F}), S_{k}\right)$ the dual basis. Because $i$ becomes an isomorphism after inverting $s^{0}$, we can find $\tilde{e_{1}^{*}}, \ldots, e_{m}^{*} \in \operatorname{Hom}_{S_{k}}\left(\mathbb{H}_{T}^{\bullet}\left(\mathcal{F}_{X^{T}}\right), S_{k}\left[1 / s^{0}\right]\right)$ such that $e_{j}^{*}=\tilde{e}_{j}^{*} \circ i$ for $1 \leq j \leq m$. Note that $f$ is in $\mathbb{H}_{\mathrm{T}}^{\bullet}(\mathcal{F})$ if and only if $\tilde{e}_{j}^{*}(f)$ is contained in $S_{k}$ for $1 \leq j \leq m$.

By Theorem 3.2, the map

$$
\mathbb{H}_{T}^{\bullet}(\mathcal{F}) \hookrightarrow \mathbb{H}_{T}^{\bullet}\left(\mathcal{F}_{X^{\alpha}}\right)
$$

becomes an isomorphism after inverting $s^{\alpha}$. As $f$ is contained in $\mathbb{H}_{T}^{\bullet}\left(\mathcal{F}_{X^{\alpha}}\right)$, we conclude $\tilde{e}_{j}^{*}(f) \in S_{k}\left[1 / s^{\alpha}\right]$ for any $1 \leq j \leq m$. Hence,

$$
\tilde{e_{j}^{*}}(f) \in \bigcap_{\alpha \in X^{*}(T)} S_{k}\left[1 / s^{\alpha}\right] .
$$

But $\bigcap_{\alpha \in X^{*}(T)} S_{k}\left[1 / s^{\alpha}\right]=S_{k}$ as the greatest common divisor of all $s^{\alpha}$ is 1. Hence $\tilde{e}_{j}^{*}(f) \in S_{k}$, which is what we wanted to show.

\section{EqUiVARIANT PARITY SHEAVES}

In the following sections we consider equivariant parity sheaves on a stratified variety, which were introduced in [JMW09a]. It turns out that the equivariant cohomology of such a sheaf is free over the symmetric algebra, so by the results in the previous sections it can be calculated by moment graph techniques. We determine the corresponding sheaves on the moment graph explicitely: we show that these are the sheaves that are constructed by the Braden-MacPherson algorithm (cf. [BM01]).

For all of the above, we need an additional datum: a stratification of the variety. 
5.1. Stratified varieties. We assume from now that the $T$-variety $X$ is endowed with a stratification

$$
X=\bigsqcup_{\lambda \in \Lambda} X_{\lambda}
$$

by $T$-stable subvarieties $X_{\lambda}$. We write $D_{T, \Lambda}^{b}(X, k)$ for the full subcategory of $D_{T}^{b}(X, k)$ consisting of objects which are constructible with respect to this stratification. In addition to the assumptions (A1) and (A2) we assume:

(S1) For each $\lambda \in \Lambda$ there is a $T$-equivariant isomorphism $X_{\lambda} \cong \mathbb{C}^{d_{\lambda}}$, where $\mathbb{C}^{d_{\lambda}}$ carries a linear $T$-action.

(S2) The category $D_{T, \Lambda}^{b}(X, k)$ is preserved by under GrothendieckVerdier duality. (This is satisfied, for example, if the stratification is Whitney.)

By (A1) and (A2) each stratum $X_{\lambda}$ contains a unique fixed point. We denote this fixed point by $x_{\lambda}$.

The topology of $X$ gives us a partial order on the set $\Lambda$ : We set $\lambda \leq \mu$ if and only if $X_{\lambda} \subset \bar{X}_{\mu}$. We use the following notation for an arbitrary partially ordered set $\Lambda$ : For $\lambda \in \Lambda$ we set $\{\geq \lambda\}:=\{\nu \in \Lambda \mid \nu \geq \lambda\}$ and we define $\{\leq \lambda\},\{>\lambda\}$, etc. in an analogous fashion.

Definition 5.1. Let $\mathcal{K}$ be a subset of $\Lambda$.

- We say that $\mathcal{K}$ is open, if for all $\gamma \in \mathcal{K}, \lambda \in \Lambda$ with $\lambda \geq \gamma$ we have $\lambda \in \mathcal{K}$, i.e. if $\mathcal{K}=\bigcup_{\gamma \in \mathcal{K}}\{\geq \gamma\}$.

- We say that $\mathcal{K}$ is closed if $\Lambda \backslash \mathcal{K}$ is open, i.e. if $\mathcal{K}=\bigcup_{\gamma \in \mathcal{K}}\{\leq \gamma\}$.

- We say that $\mathcal{K}$ is locally closed if it is the intersection of an open and a closed subset of $\Lambda$.

For a subset $\mathcal{K}$ of $\Lambda$ the set $\mathcal{K}^{+}:=\bigcup_{\gamma \in \mathcal{K}}\{\geq \gamma\}$ is the smallest open subset containing $\mathcal{K}$, and $\mathcal{K}^{-}:=\bigcup_{\gamma \in \mathcal{K}}\{\leq \gamma\}$ is the smallest closed subset containing $\mathcal{K}$. $\mathcal{K}$ is locally closed if $\mathcal{K}=\mathcal{K}^{-} \cap \mathcal{K}^{+}$.

For any subset $\mathcal{K}$ of $\Lambda$ we define

$$
X_{\mathcal{K}}=\bigsqcup_{\gamma \in \mathcal{K}} X_{\gamma} \subset X
$$

If $\mathcal{K}$ is open (closed, locally closed), then $X_{\mathcal{K}}$ is an open (closed, locally closed, resp.) subvariety in $X$. In particular, for any $\lambda \in \Lambda$ the subvariety $X_{\leq \lambda}:=X_{\{\leq \lambda\}}$ is closed. For $\mathcal{F} \in D_{T, \Lambda}^{b}(X, k)$ we define $\mathcal{F}_{\mathcal{K}}:=\mathcal{F}_{X_{\mathcal{K}}}$.

5.2. Equivariant parity sheaves. For $\lambda \in \Lambda$ we denote by $i_{\lambda}: X_{\lambda} \rightarrow$ $X$ the inclusion. We now give the definition of an equivariant parity sheaf on $X$ : 
Definition 5.2. Let? either denote the symbol $*$ or the symbol!, and let $\mathcal{P} \in D_{T}^{b}(X, k)$.

- $\mathcal{P}$ is ?-even (resp. ?-odd) if for all $\lambda \in \Lambda$ the sheaf $i_{\lambda}^{?} \mathcal{P}$ is a direct sum of constant sheaves appearing only in even (resp. odd) degrees.

- $\mathcal{P}$ is even (resp. odd) if it is both *-even and!-even (both $*$-odd and!-odd, resp.).

- $\mathcal{P}$ is parity if it may be written as a sum $\mathcal{P}=\mathcal{P}_{0} \oplus \mathcal{P}_{1}$ with $\mathcal{P}_{0}$ even and $\mathcal{P}_{1}$ odd.

Note that, by assumption (S1), for all $\lambda \in \Lambda$, all $T$-equivariant local systems on $X_{\lambda}$ are trivial and we have

$$
H_{T}^{\bullet}\left(X_{\lambda}, k\right)=H_{T}^{\bullet}(p t, k)=S_{k} .
$$

Hence, we have the following classification of indecomposable parity sheaves (see [JMW09a, Theorem 2.9]):

Theorem 5.3. Suppose that $k$ is a complete local ring. For all $\lambda \in \Lambda$ there exists, up to isomorphism, at most one indecomposable parity sheaf $\mathcal{P}(\lambda)$ extending the equivariant constant sheaf $\underline{k}_{X_{\lambda}}$. Moreover, any indecomposable parity sheaf is isomorphic to $\mathcal{P}(\lambda)[i]$ for some $\lambda \in \Lambda$ and some integer $i$.

Note that in this paper (in contrast to [JMW09a]) we normalise indecomposable parity sheaves in such a way that the restriction of $\mathcal{P}(\lambda)$ to $X_{\lambda}$ is the constant sheaf in degree 0. Also, in [JMW09a] parity sheaves a considered with respect to an arbitrary "pariversity" $\dagger: \Lambda \rightarrow \mathbb{Z} / 2 \mathbb{Z}$. In this paper we only consider parity sheaves with respect to the constant pariversity, which corresponds to the above definition.

Proposition 5.4. Let $\lambda \in \Lambda$ and assume that $\mathcal{P}(\lambda)$ exists. We have $\mathbb{D}(\mathcal{P}(\lambda)) \cong \mathcal{P}(\lambda)\left[2 d_{\lambda}\right]$ where $d_{\lambda}$ denotes the complex dimension of $X_{\lambda}$.

Proof. This is a simple consequence of the above theorem, together with the fact that $\mathbb{D}$ preserves parity and the fact that $\mathbb{D} \underline{k}_{X_{\lambda}} \cong \underline{k}_{X_{\lambda}}\left[2 d_{\lambda}\right]$.

5.3. Short exact sequences involving parity sheaves. Let $\mathcal{Q}$ be a parity sheaf on $X$ and let $\mathcal{J} \subset \Lambda$ be an open subset with closed complement $\mathcal{I}=\Lambda \backslash \mathcal{J}$. Denote by $j: X_{\mathcal{J}} \rightarrow X$ and $i: X_{\mathcal{I}} \rightarrow X$ the corresponding inclusions. Consider the distinguished triangle

$$
i_{i} i^{!} \mathcal{Q} \rightarrow \mathcal{Q} \rightarrow j_{*} j^{*} \mathcal{Q} \stackrel{[1]}{\rightarrow} .
$$


Lemma 5.5. (1) The above triangle gives rise to a short exact sequence

$$
0 \rightarrow \mathbb{H}_{\mathrm{T}}^{\bullet}\left(i^{!} \mathcal{Q}\right) \rightarrow \mathbb{H}_{\mathrm{T}}^{\bullet}(\mathcal{Q}) \rightarrow \mathbb{H}_{\mathrm{T}}^{\bullet}\left(\mathcal{Q}_{\mathcal{J}}\right) \rightarrow 0
$$

(2) Let $\mathcal{P}$ be another parity sheaf on $X$. Then the above triangle gives rise to a short exact sequence

$$
0 \rightarrow \operatorname{Hom}^{\bullet}\left(i^{*} \mathcal{P}, i^{!} \mathcal{Q}\right) \rightarrow \operatorname{Hom}^{\bullet}(\mathcal{P}, \mathcal{Q}) \rightarrow \operatorname{Hom}^{\bullet}\left(\mathcal{P}_{\mathcal{J}}, \mathcal{Q}_{\mathcal{J}}\right) \rightarrow 0
$$

Proof. We may assume without loss of generality that $\mathcal{Q}$ is even. Then the distinguished triangle in (3) is a distinguished triangle of !-even sheaves. If $\mathcal{P}$ (resp. $\mathcal{Q}^{\prime}$ ) is $*$-even (resp. !-even) then an induction (see [JMW09a, Corollary 2.8]) shows that $\operatorname{Hom}\left(\mathcal{P}, \mathcal{Q}^{\prime}[n]\right)=0$ for odd $n$. Then (2) follows and part (1) is the case $\mathcal{P}=\underline{k}_{X}$.

5.4. Further properties of equivariant parity sheaves. The following properties of the equivariant cohomology of parity sheaves will be useful when we come to relate parity sheaves and Braden-MacPherson sheaves in the next section.

Proposition 5.6. Suppose that $\mathcal{P}$ is an equivariant parity sheaf on $X$. Then the following holds:

(1) For any open subset $\mathcal{J}$ of $\Lambda$ the equivariant cohomology $\mathbb{H}_{\mathrm{T}}^{\bullet}\left(\mathcal{P}_{\mathcal{J}}\right)$ is a free $S_{k}$-module.

(2) For any open subset $\mathcal{J}$ of $\Lambda$ the restriction homomorphism $\mathbb{H}_{\mathrm{T}}^{\bullet}(\mathcal{P}) \rightarrow \mathbb{H}_{\mathrm{T}}^{\bullet}\left(\mathcal{P}_{\mathcal{J}}\right)$ is surjective.

(3) Assume that (A4b) holds and suppose that $E \subset X_{\lambda}$ is a onedimensional $T$-orbit. Then the restriction map

$$
\rho_{\lambda, E}: \mathbb{H}_{\mathrm{T}}^{\bullet}\left(\mathcal{P}_{x_{\lambda}}\right) \rightarrow \mathbb{H}_{\mathrm{T}}^{\bullet}\left(\mathcal{P}_{E}\right)
$$

is surjective with kernel $\alpha_{E} \mathbb{H}_{\mathrm{T}}^{\bullet}\left(\mathcal{P}_{x_{\lambda}}\right)$.

Proof. Note that (2) has already been shown in the previous lemma. For (1), first note that if we choose an open subset $\mathcal{J} \subset \Lambda$ then $\mathcal{P}_{\mathcal{J}}$ is a parity sheaf on $X_{\mathcal{J}}$. Hence it is enough to show that $\mathbb{H}_{\mathrm{T}}^{\bullet}(\mathcal{P})$ is a free $S_{k}$-module. Choose $x \in \Lambda$ minimal, let $\mathcal{I}=\{x\}$ and $\mathcal{J}=\Lambda \backslash\{x\}$. We have an exact sequence

$$
0 \rightarrow \mathbb{H}_{\mathrm{T}}^{\bullet}\left(i^{!} \mathcal{P}\right) \rightarrow \mathbb{H}_{\mathrm{T}}^{\bullet}(\mathcal{P}) \rightarrow \mathbb{H}_{\mathrm{T}}^{\bullet}\left(\mathcal{P}_{\mathcal{J}}\right) \rightarrow 0
$$

As $\mathcal{P}$ is a parity sheaf, $i ! \mathcal{P}$ is a direct sum of constant sheaves and so $\mathbb{H}_{\mathrm{T}}^{\bullet}(i ! \mathcal{P})$ is a free $S_{k}$-module. Using induction we can assume that $\mathbb{H}_{\mathrm{T}}^{\bullet}\left(\mathcal{P}_{\mathcal{J}}\right)$ is a free $S_{k}$-module. Hence $\mathbb{H}_{\mathrm{T}}^{\bullet}(\mathcal{P})$ is free.

Let us prove (3). Since $E \cup\left\{x_{\lambda}\right\}$ is contained in $X_{\lambda}$, the restriction of $\mathcal{P}$ to $E \cup\left\{x_{\lambda}\right\}$ is isomorphic to a sum of shifted constant sheaves. Hence it is enough to show that if $T$ acts on $\mathbb{C}$ via the character $\alpha \neq 0$ 
such that $n$ is invertible in $k$ if $\alpha$ is divisible by $n$ in $X^{*}(T)$, then the map

$$
\rho_{0, \mathbb{C} \times}: H_{T}^{\bullet}(\{0\}, k) \rightarrow H_{T}^{\bullet}\left(\mathbb{C}^{\times}, k\right)
$$

identifies with the canonical quotient map $S_{k} \rightarrow S_{k} / \alpha S_{k}$. With characteristic 0 coefficients this is proved in [Jan09, Section 1.10]. The divisibility assumption guarantees that the argument given there also works with coefficients in $k$.

5.5. Obtaining parity sheaves via resolutions. Up until now we have only discussed various properties of parity sheaves, without discussing their existence. We now show that the existence of certain proper morphisms to the varieties $\overline{X_{\lambda}}$ guarantees the existence of parity sheaves.

Assume that, for all $\lambda \in \Lambda$, there exists a $T$-variety $\widetilde{X_{\lambda}}$ and a proper surjective morphism

$$
\pi_{\lambda}: \widetilde{X_{\lambda}} \rightarrow \overline{X_{\lambda}}
$$

such that:

(R1) each $\widetilde{X_{\lambda}}$ is smooth and admits a $T$-equivariant closed embedding $\widetilde{X_{\lambda}} \hookrightarrow \mathbb{P}(V)$ for some $T$-module $V$,

(R2) the fixed point set $\widetilde{X}_{\lambda}^{T}$ is finite,

(R3) $\pi_{\lambda *} \underline{k}_{\widetilde{X_{\lambda}}}$ is constructible with respect to the stratification $\Lambda$ (that is, $\left.\pi_{\lambda * \underline{k}_{\widetilde{X_{\lambda}}}} \in D_{T, \Lambda}^{b}(X, k)\right)$.

Note that we do not assume that the morphisms $\pi_{\lambda}$ are birational.

Theorem 5.7. Assume that $k$ is a complete local principal ideal domain. With the above assumptions we have:

(1) For all $\lambda \in \Lambda$ there exists an indecomposable parity sheaf $\mathcal{P}(\lambda) \in$ $D_{T}^{b}(X, k)$ with support equal to $\overline{X_{\lambda}}$ and such that $\mathcal{P}(\lambda)_{X_{\lambda}} \cong \underline{k}_{X_{\lambda}}$.

(2) For all $\mu \leq \lambda$ the restriction homomorphism

$$
\mathbb{H}_{\mathrm{T}}^{\bullet}(\mathcal{P}(\lambda)) \rightarrow \mathbb{H}_{\mathrm{T}}^{\bullet}\left(\mathcal{P}(\lambda)_{x_{\mu}}\right)
$$

is surjective.

(3) The cohomology $\mathbb{H}_{\mathrm{T}}^{\bullet}(\mathcal{P}(\lambda))$ is self-dual of degree $2 \operatorname{dim} X_{\lambda}$. That is,

$$
\operatorname{Hom}_{S_{k}}^{\bullet}\left(\mathbb{H}_{\mathrm{T}}^{\bullet}(\mathcal{P}(\lambda)), S_{k}\right) \cong \mathbb{H}_{\mathrm{T}}^{\bullet}(\mathcal{P}(\lambda))\left[2 \operatorname{dim} X_{\lambda}\right]
$$

Before proving the theorem we state and prove two propositions. For this we need some more notation. Given a $T$-variety $Z$ we write $\omega_{Z}$ for the $T$-equivariant dualising sheaf in $D_{T}^{b}(Z, k)$. Note that, up to reindexing, $\mathbb{H}_{\mathrm{T}}^{\bullet}\left(\omega_{Z}\right)$ is the $T$-equivariant Borel-Moore homology of $Z$.

Let us fix $\mu \leq \lambda$ and set $F:=\pi_{\lambda}^{-1}\left(x_{\mu}\right)$. We have: 
Proposition 5.8. (1) $\mathbb{H}_{\mathrm{T}}^{\bullet}\left(\omega_{F}\right)$ and $\mathbb{H}_{\mathrm{T}}^{\bullet}\left(\omega_{\widetilde{X_{\lambda}}}\right)$ are free $S_{k}$-modules concentrated in even degrees.

(2) The canonical map $\mathbb{H}_{\mathrm{T}}^{\bullet}\left(\omega_{F}\right) \rightarrow \mathbb{H}_{\mathrm{T}}^{\bullet}\left(\omega_{\widetilde{X_{\lambda}}}\right)$ is a split injection of $S_{k}$-modules.

Proof. As $x_{\mu}$ is attractive, there exists a one parameter subgroup $\gamma: \mathbb{C}^{\times} \rightarrow$ $T$ which contracts an open neighbourhood of $x_{\mu}$ in $X$ onto $x_{\mu}$ as $z \in \mathbb{C}^{\times}$ goes to 0. Moreover, we can choose $\gamma$ such that $\widetilde{X}_{\lambda}{ }^{\mathbb{C}^{\times}}={\widetilde{X_{\lambda}}}^{T}$.

Now consider the Bialynicki-Birula's minus decomposition of $\widetilde{X}_{\lambda}{ }^{T}$ with respect to $\gamma$. That is, for each $x \in \widetilde{X}_{\lambda}^{T}$ set

$$
Y_{x}^{-}:=\left\{y \in \widetilde{X_{\lambda}} \mid \lim _{z \rightarrow \infty} \gamma(z) \cdot y=x\right\}
$$

Then a theorem of Bialynicki-Birula $([\mathrm{BB} 81])$ asserts that each $Y_{x}^{-}$is a locally closed $T$-stable subvariety of $\widetilde{X_{\lambda}}$ isomorphic to an affine space. Our choice of $\gamma$ implies that

$$
F=\pi_{\lambda}^{-1}\left(x_{\mu}\right)=\bigsqcup_{x \in F^{T}} Y_{x}
$$

Moreover, by assumption we can find a $T$-equivariant embedding

$$
\widetilde{X_{\lambda}} \hookrightarrow \mathbb{P}(V)
$$

and we may decompose $V \cong \bigoplus V_{i}$ where $V_{i}$ denotes the $i^{\text {th }}$ weight space of the $\mathbb{C}^{*}$-action on $V$ given by $\gamma$. If we set $V_{\leq i}=\bigoplus_{j \leq i} V_{i}$ then it is straightforward to check that the filtration

$$
\emptyset \subset \cdots \subset \mathbb{P}\left(V_{\leq i}\right) \subset \mathbb{P}\left(V_{\leq i+1}\right) \subset \cdots \subset \mathbb{P}(V)
$$

induces filtrations of $\widetilde{X_{\lambda}}$ and $F$ by closed subvarieties such that each successive difference is a disjoint union of Bialynicki-Birula cells. A simple induction (see, for example, [Ful97] for the non-equivariant case) shows that both $\mathbb{H}_{\mathrm{T}}^{\bullet}\left(\omega_{\widetilde{X_{\lambda}}}\right)$ and $\mathbb{H}_{\mathrm{T}}^{\bullet}\left(\omega_{F}\right)$ are free $S_{k}$-modules with basis given by the equivariant fundamental classes of closures of the Bialynicki-Birula cells. The two statements of the lemma then follow.

Proposition 5.9. With notation as above we have:

(1) $\pi_{\lambda * \underline{k}_{\widetilde{X_{\lambda}}}}$ is parity and its support is equal to $\overline{X_{\lambda}}$.

(2) For all $\mu \leq \lambda$ the restriction homomorphism

$$
\mathbb{H}_{\mathrm{T}}^{\bullet}\left(\pi_{\lambda *} \underline{k}_{\widetilde{X_{\lambda}}}\right) \rightarrow \mathbb{H}_{\mathrm{T}}^{\bullet}\left(\left(\pi_{\lambda *} \underline{k}_{\widetilde{X_{\lambda}}}\right)_{x_{\mu}}\right)
$$

is surjective. 
Proof. The support claim follows from the surjectivity of $\pi_{\lambda}$. We now

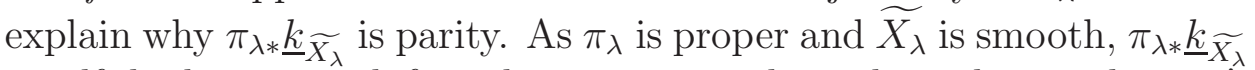
is self-dual up to a shift and so it is enough to show that $\pi_{\lambda *} \underline{k}_{\widetilde{X_{\lambda}}}$ is !even. As $\pi_{\lambda *} \underline{k}_{\widetilde{X_{\lambda}}}$ is constructible with respect to the $\Lambda$-stratification, it is enough to show that, for all $\mu, i_{x_{\mu}}^{!} \pi_{\lambda *} \underline{k}_{\widetilde{X_{\lambda}}}$ is a direct sum of constant sheaves concentrated in even degrees, where $i_{x_{\mu}}$ denotes the inclusion $\left\{x_{\mu}\right\} \hookrightarrow X$. A devissage argument shows that this is the case if and only if $\mathbb{H}_{\mathrm{T}}^{\bullet}\left(i_{x_{\mu}}^{!} \pi_{\lambda * \underline{k}}{\underline{\widetilde{X_{\lambda}}}}\right)$ is a free $S_{k^{-}}$-module.

By proper base change $i_{x_{\mu}}^{!} \pi_{\lambda * \underline{k}_{\widetilde{X_{\lambda}}}}$ is isomorphic (up to a shift) to $\pi_{\lambda *} \omega_{F}$. Hence it is enough to show that $\mathbb{H}_{\mathrm{T}}^{\bullet}\left(\omega_{F}\right)$ is a free $S_{k}$-module concentrated in even degrees. This is the case by Proposition 5.8(1) above.

For the second statement of the proposition note that the restriction homomorphism $\mathbb{H}_{\mathrm{T}}^{\bullet}\left(\pi_{\lambda *}{\underline{\underline{X_{\lambda}}}}_{\widetilde{1}}\right) \rightarrow \mathbb{H}_{\mathrm{T}}^{\bullet}\left(\left(\pi_{\lambda *}{\underline{\underline{X_{\lambda}}}}_{\widetilde{x}_{\mu}}\right)\right.$ is dual (again, up to a shift) to the canonical map $\mathbb{H}_{\mathrm{T}}^{\bullet}\left(\omega_{F}\right) \rightarrow \mathbb{H}_{\mathrm{T}}^{\bullet}\left(\omega_{\widetilde{X_{\lambda}}}\right)$ which is a split injection by Proposition 5.8(2).

Proof of Theorem 5.7. By Proposition 5.9, $\pi_{\lambda * \underline{k}_{\widetilde{X_{\lambda}}}} \in D_{T}^{b}(X, k)$ is parity. If we let $\mathcal{Q}$ denote an indecomposable summand of $\pi_{\lambda *} \underline{k}_{\widetilde{X_{\lambda}}}$ containing $X_{\lambda}$ in its support then $\mathcal{Q}$ is also parity and, by Theorem 5.3, we have $\mathcal{Q}_{X_{\lambda}} \cong \underline{k}_{X_{\lambda}}[i]$ for some integer $i$. It follows that we can take $\mathcal{P}(\lambda):=\mathcal{Q}[-i]$.

Another consequence of Theorem 5.3 is that any indecomposable parity sheaf $\mathcal{P}(\lambda)$ occurs as a direct summand of $\pi_{\lambda *} \underline{k}_{\widetilde{X_{\lambda}}}[i] \in D_{T}^{b}(X, k)$ for some $i$. Hence, to show Part (2) of the theorem it is enough to check that the map

$$
\mathbb{H}_{\mathrm{T}}^{\bullet}\left(\pi_{\lambda *}{\underline{\underline{X_{\lambda}}}}\right) \rightarrow \mathbb{H}_{\mathrm{T}}^{\bullet}\left(\left(\pi_{\lambda * \underline{k}}{\underline{\widetilde{X_{\lambda}}}}_{x_{\mu}}\right)\right.
$$

is surjective. This is the case by Proposition 5.9.

By Proposition 5.4 we have $\mathbb{D} \mathcal{P}(\lambda) \cong \mathcal{P}(\lambda)\left[2 \operatorname{dim} X_{\lambda}\right]$. We also know that $\mathbb{H}_{\mathrm{T}}^{\bullet}(\mathcal{P}(\lambda))$ is a free $S_{k}$-module by Proposition 5.8 (recall that $\omega_{\widetilde{X_{\lambda}}} \cong$ $\underline{k}_{\widetilde{X_{\lambda}}}$ because $\widetilde{X_{\lambda}}$ is smooth). Hence

$$
\operatorname{Hom}_{S_{k}}^{\bullet}\left(\mathbb{H}_{\mathrm{T}}^{\bullet}(\mathcal{P}(\lambda)), S_{k}\right) \cong \mathbb{H}_{\mathrm{T}}^{\bullet}(\mathcal{P}(\lambda))\left[2 \operatorname{dim} X_{\lambda}\right]
$$

as $\overline{X_{\lambda}}$ is proper.

5.6. Parity sheaves and the functor $\mathbb{W}$. In this section we begin discussing the relationship between parity sheaves and the localisation functor $\mathbb{W}$. In particular, we show that $\mathbb{W}$ is fully faithful on morphisms of all degrees between parity sheaves.

For the rest of this section we assume (A1)-(A4a/b) and (S1), (S2). 
Proposition 5.10. Let $\mathcal{P}(\lambda)$ be a parity sheaf. Then the localisation homomorphism $\mathbb{H}_{\mathrm{T}}^{\bullet}(\mathcal{P}(\lambda)) \rightarrow \mathbb{H}_{\mathrm{T}}^{\bullet}\left(\mathcal{P}(\lambda)_{X^{T}}\right)$ identifies $\mathbb{H}_{\mathrm{T}}^{\bullet}(\mathcal{P}(\lambda))$ with the global sections of $\mathbb{W}(\mathcal{P}(\lambda))$.

Proof. In order to apply Theorem 4.4 we only need to check that $\mathbb{H}_{\mathrm{T}}^{\bullet}(\mathcal{P}(\lambda))$ and $\mathbb{H}_{\mathrm{T}}^{\bullet}\left(\mathcal{P}(\lambda)_{X^{T}}\right)$ are free $S_{k^{-}}$-modules. This is the case for $\mathbb{H}_{\mathrm{T}}^{\bullet}(\mathcal{P}(\lambda))$ by Proposition 5.6. For $\mathbb{H}_{\mathrm{T}}^{\bullet}\left(\mathcal{P}(\lambda)_{X^{T}}\right)$ note that because $\mathcal{P}(\lambda)$ is parity, the restriction of $\mathcal{P}(\lambda)$ to any $T$-fixed point is a direct sum of equivariant constant sheaves.

Theorem 5.11. The functor $\mathbb{W}$ is fully faithful when restricted to parity sheaves, i.e. if $\mathcal{P}$ and $\mathcal{P}^{\prime}$ are parity sheaves on $X$, then

$$
\operatorname{Hom}_{D_{T}^{b}(X, k)}^{\bullet}\left(\mathcal{P}, \mathcal{P}^{\prime}\right) \rightarrow \operatorname{Hom}_{\mathcal{G} \text { - } \bmod _{k}^{\mathbb{Z}}}^{\bullet}\left(\mathbb{W}(\mathcal{P}), \mathbb{W}\left(\mathcal{P}^{\prime}\right)\right)
$$

is an isomorphism.

Proof. Without loss of generality we can assume that both $\mathcal{P}$ and $\mathcal{P}^{\prime}$ are even. Let $\lambda \in \Lambda$ be a minimal element and set $\mathcal{J}=\Lambda \backslash\{\lambda\}$. Denote by $j: X_{\mathcal{J}}=X \backslash X_{\lambda} \rightarrow X$ the corresponding open inclusion and by $i: X_{\lambda} \rightarrow X$ the complementary closed inclusion. Then we have a distinguished triangle

$$
i_{i} i^{\prime} \mathcal{P}^{\prime} \rightarrow \mathcal{P}^{\prime} \rightarrow j_{*} j^{*} \mathcal{P}^{\prime} \stackrel{[1]}{\rightarrow}
$$

which gives rise, by Lemma 5.5, to a short exact sequence

$$
0 \rightarrow \operatorname{Hom}^{\bullet}\left(i^{*} \mathcal{P}, i^{!} \mathcal{P}^{\prime}\right) \rightarrow \operatorname{Hom}^{\bullet}\left(\mathcal{P}, \mathcal{P}^{\prime}\right) \rightarrow \operatorname{Hom}^{\bullet}\left(\mathcal{P}_{\mathcal{J}}, \mathcal{P}_{\mathcal{J}}^{\prime}\right) \rightarrow 0
$$

of graded spaces. The map $\operatorname{Hom}^{\bullet}\left(\mathcal{P}, \mathcal{P}^{\prime}\right) \rightarrow \operatorname{Hom}^{\bullet}\left(\mathcal{P}_{\mathcal{J}}, \mathcal{P}_{\mathcal{J}}^{\prime}\right)$ is induced by the restriction to an open subspace, hence we can fit the above short exact sequence into a commutative diagram

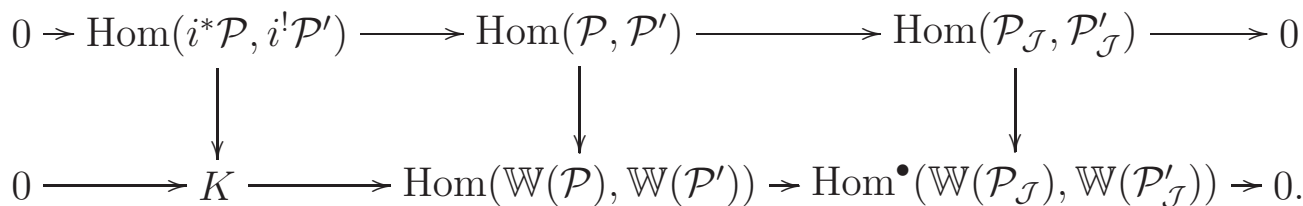

As $\mathcal{P}_{\mathcal{J}}$ and $\mathcal{P}_{\mathcal{J}}^{\prime}$ are parity sheaves on $X_{\mathcal{J}}$ we can, by induction on the number of strata, assume that the vertical map on the right is an isomorphism. Hence we can finish the proof by showing that the vertical map on the left is an isomorphism as well.

Now $K$ is the space of all morphisms $f: \mathbb{W}(\mathcal{P}) \rightarrow \mathbb{W}\left(\mathcal{P}^{\prime}\right)$ with $f^{\mu}=0$ and $f^{E}=0$ for vertices $\mu$ and edges $E$ of $\mathcal{J}$. By Proposition 5.6, (3), it identifies with the set of homomorphisms from the stalk $\mathbb{W}(\mathcal{P})^{\lambda}$ into the costalk $\mathbb{W}\left(\mathcal{P}^{\prime}\right)_{\lambda}$. By definition we have $\mathbb{W}(\mathcal{P})^{\lambda}=\mathbb{H}_{\mathrm{T}}^{\bullet}\left(i^{*} \mathcal{P}\right)$. Now let us look at the short exact sequence

$$
0 \rightarrow \mathbb{H}_{\mathrm{T}}^{\bullet}\left(i^{!} \mathcal{P}^{\prime}\right) \rightarrow \mathbb{H}_{\mathrm{T}}^{\bullet}\left(\mathcal{P}^{\prime}\right) \rightarrow \mathbb{H}_{\mathrm{T}}^{\bullet}\left(\mathcal{P}_{\mathcal{J}}^{\prime}\right) \rightarrow 0
$$


given by Lemma 5.5. By Proposition 5.10, $\mathbb{H}_{\mathrm{T}}^{\bullet}\left(\mathcal{P}^{\prime}\right)$ and $\mathbb{H}_{\mathrm{T}}^{\bullet}\left(\mathcal{P}_{\mathcal{J}}^{\prime}\right)$ can be identified with the sections of $\mathbb{W}\left(\mathcal{P}^{\prime}\right)$ over $\Lambda$ and $\mathcal{J}$, respectively. Hence we may identify

$$
\mathbb{H}_{\mathrm{T}}^{\bullet}\left(i^{!} \mathcal{P}^{\prime}\right)=\mathbb{W}\left(\mathcal{P}^{\prime}\right)_{\lambda}
$$

As $i^{*} \mathcal{P}$ and $i ! \mathcal{P}^{\prime}$ are free sheaves on $X_{\lambda}$, we deduce from the above that the homomorphism $\operatorname{Hom}\left(i^{*} \mathcal{P}, i^{!} \mathcal{P}^{\prime}\right) \rightarrow K$ in the commutative diagram above is an isomorphism as well.

\section{Braden-MacPherson Sheaves on a MOMEnT GRAPH}

We return now to the theory of sheaves on a moment graph. We first motivate the definition of the Braden-MacPherson sheaves by considering the problem of extending local sections. Then we prove one of our main results, namely that the functor $\mathbb{W}$ sends parity sheaves to Braden-MacPherson sheaves.

6.1. Extending local sections. Let $\mathcal{G}=(\mathcal{V}, \mathcal{E}, \alpha)$ be a moment graph. Suppose that each edge is given a direction. Then, for $x, y \in \mathcal{V}$, we set $x \leqslant y$ if and only if there is a directed path from $x$ to $y$. We assume that this determines a partial order on $\mathcal{V}$, i.e. we assume that there are no non-trivial closed directed paths. We call this datum a directed moment graph.

Recall that we call a subset $\mathcal{J}$ of $\mathcal{V}$ open if it contains with any element all elements that are larger in the partial order, i.e. all elements that can be reached by a directed path. For a sheaf $\mathscr{M}$ and an open subset $\mathcal{J}$ of $\mathcal{V}$ we call an element in $\Gamma(\mathcal{J}, \mathscr{M})$ a local section.

Now we want to find some conditions on $\mathscr{M}$ that ensure that each local section can be extended to a global section, i.e. which ensure that the restriction $\Gamma(\mathscr{M}) \rightarrow \Gamma(\mathcal{J}, \mathscr{M})$ from global to local sections is surjective for any open set $\mathcal{J}$. For this we need the following definitions.

For a vertex $x$ of $\mathcal{G}$ we define

$$
\mathcal{V}_{\delta x}:=\{y \in \mathcal{V} \mid \text { there is an edge } E: x \rightarrow y\} .
$$

So $\mathcal{V}_{\delta x}$ is the subset of vertices $y$ that are bigger than $x$ in the partial order and that are connected to $x$ by an edge. We denote by

$$
\mathcal{E}_{\delta x}:=\{E \in \mathcal{E} \mid E: x \rightarrow y\}
$$

the set of the corresponding edges. Then there is an obvious correspondence $\mathcal{E}_{\delta x} \stackrel{\sim}{\rightarrow} \mathcal{V}_{\delta x}$ (as we assume that two vertices are connected by at most one edge). For a sheaf $\mathscr{M}$ and a vertex $x$ we define the map

$$
u_{x}: \Gamma(\{>x\}, \mathscr{M}) \rightarrow \bigoplus_{E \in \mathcal{E}_{\delta x}} \mathscr{M}^{E}
$$


as the composition

$$
\Gamma(\{>x\}, \mathscr{M}) \subset \bigoplus_{y>x} \mathscr{M}^{y} \stackrel{p}{\rightarrow} \bigoplus_{y \in \mathcal{V}_{\delta x}} \mathscr{M}^{y} \stackrel{\rho}{\rightarrow} \bigoplus_{E \in \mathcal{E}_{\delta x}} \mathscr{M}^{E}
$$

where $p$ is the projection along the decomposition and $\rho=\bigoplus_{y \in \mathcal{V}_{\delta x}} \rho_{y, E}$. We let

$$
\mathscr{M}^{\delta x}:=u_{x}(\Gamma(\{>x\}, \mathscr{M})) \subset \bigoplus_{E \in \mathcal{E}_{\delta x}} \mathscr{M}^{E}
$$

be the image of this map. Moreover, we define the map

$$
d_{x}:=\left(\rho_{x, E}\right)_{E \in \mathcal{E}_{\delta x}}^{T}: \mathscr{M}^{x} \rightarrow \bigoplus_{E \in \mathcal{E}_{\delta x}} \mathscr{M}^{E} .
$$

The connection of the above definitions with the problem of extending local sections is the following. Suppose that $m^{\prime} \in \Gamma(\{>x\}, \mathscr{M})$ is a section and that $m_{x} \in \mathscr{M}^{x}$. Then the concatenated element $\left(m_{x}, m^{\prime}\right) \in$ $\bigoplus_{y \geq x} \mathscr{M}^{y}$ is a section over $\{\geq x\}$ if and only if $u_{x}\left(m^{\prime}\right)=d_{x}\left(m_{x}\right)$.

Lemma 6.1. For a sheaf $\mathscr{M}$ on the moment graph $\mathcal{G}$ the following are equivalent:

(1) For any open subsets $\mathcal{J}^{\prime} \subset \mathcal{J}$ of $\mathcal{V}$ the restriction map $\Gamma(\mathcal{J}, \mathscr{M}) \rightarrow$ $\Gamma\left(\mathcal{J}^{\prime}, \mathscr{M}\right)$ is surjective.

(2) For any vertex $x \in \mathcal{V}$, the restriction map $\Gamma(\{\geq x\}, \mathscr{M}) \rightarrow$ $\Gamma(\{>x\}, \mathscr{M})$ is surjective.

(3) For any $x \in \mathcal{V}$, the map $d_{x}: \mathscr{M}^{x} \rightarrow \bigoplus_{E \in \mathcal{E}_{\delta x}} \mathscr{M}^{E}$ contains $\mathscr{M}^{\delta_{x}}$ in its image.

Proof. Clearly property (2) is a special case of property (1). Let us prove the converse, so let us assume that (2) holds. It is enough to prove property (1) in the special case that $\mathcal{J}=\mathcal{J}^{\prime} \cup\{x\}$ for a single element $x$, since we get the general case from this by induction. So let $m=\left(m_{y}\right)$ be a section in $\Gamma(\mathcal{J} \backslash\{x\}, \mathscr{M})$. Since $\{>x\} \subset \mathcal{J} \backslash\{x\}$ we can restrict $m$ and get a section $m^{\prime}$ in $\Gamma(\{>x\}, \mathscr{M})$. By assumption there is an element $m_{x} \in \mathscr{M}^{x}$ such that $\left(m_{x}, m^{\prime}\right)$ is a section over $\{\geq x\}$. As $x$ is not connected to any vertex in $\mathcal{J} \backslash\{\geq x\}$ it follows that $\left(m_{x}, m\right)$ is a section over $\mathcal{J}$. Hence (2) implies (1).

Let us show that (2) is equivalent to (3). Now (2) means that for any section $m$ over $\{>x\}$ we can find $m_{x} \in \mathscr{M}^{x}$ such that $\left(m_{x}, m\right)$ is a section over $\{\geq x\}$. But $\left(m_{x}, m\right)$ is a section over $\{\geq x\}$ if and only if $d_{x}\left(m_{x}\right)=u_{x}(m)$. Hence, a section $m$ over $\{>x\}$ can be extended to the vertex $x$ if and only of $u_{x}(m)$ is contained in the image of $d_{x}$.

For later use we prove the following statement. 
Lemma 6.2. Let $x$ be a vertex of $\mathcal{G}$ and $\mathscr{M}$ a sheaf on $\mathcal{G}$. Then the following are equivalent:

(1) The map $\Gamma(\{\geq x\}, \mathscr{M}) \rightarrow \mathscr{M}^{x}$ is surjective.

(2) The image of the map $d_{x}: \mathscr{M}^{x} \rightarrow \bigoplus_{E \in \mathcal{E}_{\delta x}} \mathscr{M}^{E}$ is contained in $\mathscr{M}^{\delta x}$.

Proof. Suppose that (1) holds and let $s \in \mathscr{M}^{x}$. Then there is a section $m$ of $\mathscr{M}$ over $\{\geq x\}$ with $m_{x}=s$. If we denote the restriction of $m$ to $\{>x\}$ by $m^{\prime}$, then this means that $d_{x}(s)=u_{x}\left(m^{\prime}\right)$. So $d_{x}(s)$ is contained in the image of $u_{x}$, which is $\mathscr{M}^{\delta x}$.

Conversely, suppose that (2) holds and let $s \in \mathscr{M}^{x}$. Then there is a section $m^{\prime}$ of $\mathscr{M}$ over $\{>x\}$ such that $d_{x}(s)=u_{x}\left(m^{\prime}\right)$. Hence $\left(s, m^{\prime}\right)$ is a section over $\{\geq x\}$, hence $s$ is contained in the image of $\Gamma(\{\geq x\}, \mathscr{M}) \rightarrow \mathscr{M}^{x}$.

6.2. Braden-MacPherson sheaves. The most important class of sheaves on a moment graph is the following.

Definition 6.3. A sheaf $\mathscr{B}$ on the moment graph $\mathcal{G}$ is called a BradenMacPherson sheaf if it satisfies the following properties:

(1) For any $x \in \mathcal{V}$, the stalk $\mathscr{B}^{x}$ is a graded free $S_{k}$-module of finite rank and only finitely many $\mathscr{B}^{x}$ are non-zero.

(2) For a directed edge $E: x \rightarrow y$ the map $\rho_{y, E}: \mathscr{B}^{y} \rightarrow \mathscr{B}^{E}$ is surjective with kernel $\alpha(E) \mathscr{B}^{y}$,

(3) For any open subset $\mathcal{J}$ of $\mathcal{V}$ the map $\Gamma(\mathscr{B}) \rightarrow \Gamma(\mathcal{J}, \mathscr{B})$ is surjective.

(4) The map $\Gamma(\mathscr{B}) \rightarrow \mathscr{B}^{x}$ is surjective for any $x \in \mathcal{V}$.

Here is a classification theorem.

Theorem 6.4. Assume that $k$ is a local ring and suppose that the moment graph is such that for any vertex $w$ the set $\{\leq w\}$ is finite. Then the following holds.

(1) For any vertex $w$ there is an up to isomorphism unique BradenMacPherson sheaf $\mathscr{B}(w)$ on $\mathcal{G}$ with the following properties:

- We have $\mathscr{B}(w)^{w} \cong S_{k}$ and $\mathscr{B}(w)^{x}=0$ unless $x \leq w$.

- $\mathscr{B}(w)$ is indecomposable in $\mathcal{G}$ - $\bmod _{k}^{\mathbb{Z}}$.

(2) Let $\mathscr{B}$ be a Braden-MacPherson sheaf. Then there are $w_{1}, \ldots, w_{n} \in$ $\mathcal{V}$ and $l_{1}, \ldots, l_{n} \in \mathbb{Z}$ such that

$$
\mathscr{B} \cong \mathscr{B}\left(w_{1}\right)\left[l_{1}\right] \oplus \cdots \oplus \mathscr{B}\left(w_{n}\right)\left[l_{n}\right] .
$$

The multiset $\left(w_{1}, l_{1}\right), \ldots,\left(w_{n}, l_{n}\right)$ is uniquely determined by $\mathscr{B}$.

Remark 1. We need the locality assumption on $k$ in order to ensure that projective covers exist in the category of graded $S_{k}$-modules. 
Proof. We first prove the existence part of statement (1). For $w \in \mathcal{V}$ we define a sheaf $\mathscr{B}(w)$ by the following inductive construction:

(1) We start with setting $\mathscr{B}(w)^{w}=S_{k}$ and $\mathscr{B}(w)^{x}=0$ if $x \not \leq w$.

(2) If we have already defined $\mathscr{B}(w)^{y}$, then we set, for each edge $E: x \rightarrow y$,

$$
\mathscr{B}(w)^{E}:=\mathscr{B}(w)^{y} / \alpha(E) \mathscr{B}(w)^{y}
$$

and we let $\rho_{y, E}: \mathscr{B}(w)^{y} \rightarrow \mathscr{B}(w)^{E}$ be the canonical map.

(3) Suppose that we have already defined $\mathscr{B}(w)^{y}$ for all $y$ in an open subset $\mathcal{J}$ and suppose that $x \in \mathcal{V}$ is such that $\mathcal{J} \cup\{x\}$ is open as well. By step (2) we have also defined the spaces $\mathscr{B}(w)^{E}$ for each edge $E: x \rightarrow y$ originating at $x$, as well as the maps $\rho_{y, E}: \mathscr{B}(w)^{y} \rightarrow \mathscr{B}(w)^{E}$. We now define $\mathscr{B}(w)^{x}$ and the maps $\rho_{x, E}$ for those edges $E$. We can already calculate the sections of $\mathscr{B}(w)$ over $\{>x\}$, as well as the $S_{k}$-modules $\mathscr{B}(w)^{\delta x} \subset$ $\bigoplus_{E \in \mathcal{E}_{\delta x}} \mathscr{B}(w)^{E}$. Now we define $d_{x}: \mathscr{B}(w)^{x} \rightarrow \mathscr{B}(w)^{\delta x}$ as a projective cover in the category of graded $S_{k}$-modules. The components of $d_{x}$ (with respect to the inclusion $\mathscr{B}(w)^{\delta x} \subset$ $\left.\bigoplus_{E \in \mathcal{E}_{\delta x}} \mathscr{B}(w)^{E}\right)$ give us the maps $\rho_{x, E}$.

Let us check that $\mathscr{B}(w)$ is indeed a Braden-MacPherson sheaf. Since $\mathscr{B}(w)^{x}$ is projective for all $x \in \mathcal{V}$ it is a graded free $S_{k}$-module and the finiteness assumptions hold as well, so $\mathscr{B}(w)$ fulfills property (1). Property (2) is assured by step (2) in the inductive construction of $\mathscr{B}(w)$. Property $(3)$ is, by Lemma 6.1 , equivalent to the fact that for all $x \in \mathcal{V}$ the map $d_{x}: \mathscr{B}(w)^{x} \rightarrow \bigoplus_{E \in \mathcal{E}_{\delta x}} \mathscr{B}(w)^{E}$ contains $\mathscr{B}(w)^{\delta_{x}}$ in its image. This is clear by step (3). In addition, step (3) also yields that the image of $d_{x}$ is contained in $\mathscr{B}(w)^{\delta x}$. By Lemma 6.2 this is equivalent to the surjectivity of $\Gamma(\{\geq x\}, \mathscr{B}(w)) \rightarrow \mathscr{B}(w)^{x}$. We have already seen that the restriction map $\Gamma(\mathscr{B}(w)) \rightarrow \Gamma(\{\geq x\}, \mathscr{B}(w))$ is surjective. Hence also $\Gamma(\mathscr{B}(w)) \rightarrow \mathscr{B}(w)^{x}$ is surjective, hence $\mathscr{B}(w)$ also satisfies property (4) of a Braden-MacPherson sheaf.

Now we prove statement (2) of the above theorem using the above explicitly defined objects $\mathscr{B}(w)$. Note that this also gives the uniqueness part of statement (1), which we have not yet proven. So let $\mathscr{B}$ be an arbitrary Braden-MacPherson sheaf. We prove by induction on the set of open subsets $\mathcal{J}$ of $\mathcal{V}$ that there are $\left(w_{1}, l_{1}\right), \ldots,\left(w_{n}, l_{n}\right)$ such that

$$
\mathscr{B}^{\mathcal{J}} \cong \mathscr{B}\left(w_{1}\right)^{\mathcal{J}}\left[l_{1}\right] \oplus \cdots \oplus \mathscr{B}\left(w_{n}\right)^{\mathcal{J}}\left[l_{n}\right]
$$

(Here and in the following we denote by $\mathscr{F}^{\mathcal{I}}$ the obvious restriction of a sheaf $\mathscr{F}$ to the subgraph corresponding to the vertices in a subset $\mathcal{I}$ of $\mathcal{V}$.) 
So suppose that $\mathcal{J}$ is open, that $x \in \mathcal{J}$ is minimal and we have a decomposition as above for $\mathcal{J}^{\prime}=\mathcal{J} \backslash\{x\}$. We get, in particular,

$$
\Gamma(\{>x\}, \mathscr{B}) \cong \Gamma\left(\{>x\}, \mathscr{B}\left(w_{1}\right)\left[l_{1}\right]\right) \oplus \cdots \oplus \Gamma\left(\{>x\}, \mathscr{B}\left(w_{n}\right)\left[l_{n}\right]\right)
$$

and

$$
\mathscr{B}^{\delta x} \cong \mathscr{B}\left(w_{1}\right)^{\delta x}\left[l_{1}\right] \oplus \cdots \oplus \mathscr{B}\left(w_{n}\right)^{\delta x}\left[l_{n}\right] .
$$

Now $d_{x}: \mathscr{B}^{x} \rightarrow \mathscr{B}^{\delta x}$ is surjective, by property (3) of a Braden-MacPherson sheaf and Lemma 6.1 , and $\bigoplus \mathscr{B}\left(w_{i}\right)^{x}\left[l_{i}\right] \rightarrow \bigoplus \mathscr{B}\left(w_{i}\right)^{\delta x}\left[l_{i}\right]$ is a projective cover by construction. Hence we have a decomposition $\mathscr{B}^{x}=$ $\bigoplus \mathscr{B}\left(w_{i}\right)^{x}\left[l_{i}\right] \oplus R$ for some graded free $S_{k}$-module $R$ which lies in the kernel of $d_{x}$. Each isomorphism $R \cong S_{k}\left[m_{1}\right] \oplus \cdots \oplus S_{k}\left[m_{r}\right]$ then yields an isomorphism

$\mathscr{B}^{\mathcal{J}} \cong \mathscr{B}\left(w_{1}\right)^{\mathcal{J}}\left[l_{1}\right] \oplus \cdots \oplus \mathscr{B}\left(w_{n}\right)^{\mathcal{J}}\left[l_{n}\right] \oplus \mathscr{B}(x)^{\mathcal{J}}\left[m_{1}\right] \oplus \cdots \oplus \mathscr{B}(x)^{\mathcal{J}}\left[m_{r}\right]$,

which is our claim for $\mathcal{J}$. The above construction also yields the uniqueness of the multiset $\left(w_{1}, l_{1}\right), \ldots,\left(w_{n}, l_{n}\right)$.

6.3. Directed moment graphs from stratified varieties. Suppose that $X$ is a complex $T$-variety satisfying (A1). In Section 4.4 we constructed an (undirected) moment graph $\mathcal{G}_{X}$ from this datum. Suppose now that, in addition, we are given a stratification $X=\bigsqcup_{\lambda \in \Lambda} X_{\lambda}$ satisfying (S1) and (S2). Recall that for each $\lambda \in \Lambda$ we denote by $x_{\lambda}$ the unique fixed point in $X_{\lambda}$. Hence we now have identifications between the set of fixed points in $X$, the set $\Lambda$ of strata and the set of vertices of $\mathcal{G}_{X}$.

From this we obtain a direction of each edge as follows. Suppose that the one-dimensional orbit $E$ contains $x_{\lambda}$ and $x_{\mu}$ in its closure. Then either $X_{\lambda} \subset \overline{X_{\mu}}$ or $X_{\mu} \subset \overline{X_{\lambda}}$. We direct the corresponding edge of $\mathcal{G}_{X}$ towards $\mu$ in the first case, and towards $\lambda$ in the second case. We denote by $\leq$ the partial order on the vertices of $\mathcal{G}_{X}$ generated by the relation $\lambda \leq \mu$ if there is an edge $E: \lambda \rightarrow \mu$. The following proposition shows that this is the same order as the one induced by the closure relations on the strata:

Proposition 6.5. We have $\lambda \leq \mu$ if and only if $X_{\lambda} \subset \overline{X_{\mu}}$.

Proof. Clearly, if $\lambda \leq \mu$ then $X_{\lambda} \subset \overline{X_{\mu}}$. For the converse we show:

If $X_{\lambda} \subset \overline{X_{\mu}}$ then there exists an edge

$E: \lambda \rightarrow \nu$ such that $X_{\nu} \subset \overline{X_{\mu}}$.

Let us assume that (4) holds. Then, if $X_{\lambda} \subset \overline{X_{\mu}}$, we can find a chain $\lambda \rightarrow \nu_{1} \rightarrow \cdots \rightarrow \nu_{m} \rightarrow \mu$ and so $\lambda \leq \mu$. 
It remains to show (4). Let $U$ be an affine $T$-stable neighbourhood of $x_{\lambda}$ in $\overline{X_{\mu}}$ and let $N_{\lambda} \subset U$ be a $T$-invariant normal slice to the stratum $X_{\lambda}$ at $x_{\lambda}$. Because $x_{\lambda} \in N_{\lambda}$ is attractive, we can find a cocharacter $\gamma: \mathbb{C}^{\times} \rightarrow T$ such that $\lim _{z \rightarrow 0} \gamma(z) \cdot x=x_{\lambda}$ for all $x \in N_{\lambda}$. It follows that $Y:=\left(N_{\lambda} \backslash\left\{x_{\lambda}\right\}\right) / \mathbb{C}^{\times}$is a projective variety. By Borel's fixed point theorem, $T$ has a fixed point on $Y$ and hence a one-dimensional orbit on $N_{\lambda}$. This one-dimensional orbit is contained in some $X_{\nu}$, hence connects $x_{\lambda}$ with $x_{\nu}$. By construction we have $X_{\nu} \subset \overline{X_{\mu}}$.

6.4. The $k$-smooth locus of a moment graph. In this subsection we assume that $k$ is a field and that the (directed) moment graph $\mathcal{G}$ contains a largest vertex $w$. This moment graph carries the indecomposable Braden-MacPherson sheaf $\mathscr{B}:=\mathscr{B}(w)$ over $k$.

Definition 6.6. The $k$-smooth locus $\Omega_{k}(\mathcal{G})$ of $\mathcal{G}$ is the set of all vertices y of $\mathcal{G}$ such that $\mathscr{B}^{y}$ is a free $S_{k}$-module of (ungraded) rank 1.

In [Fie10] the $k$-smooth locus is determined for a large class of pairs $(\mathcal{G}, k)$. In order to formulate the result, let $\mathcal{B}:=\Gamma(\mathscr{B})$ be the space of global sections of $\mathscr{B}$. We consider this as a graded $\mathcal{Z}_{k}$-module.

Definition 6.7. We say that $\mathcal{B}$ is self-dual of degree $l \in \mathbb{Z}$ if there is an isomorphism

$$
\operatorname{Hom}_{S_{k}}^{\bullet}\left(\mathcal{B}, S_{k}\right) \cong \mathcal{B}[l]
$$

of graded $\mathcal{Z}_{k}$-modules.

The following is an analogue of the assumption (A4a) for moment graphs.

Definition 6.8. We say that the pair $(\mathcal{G}, k)$ is a GKM-pair if $\alpha_{E}$ is non-zero in $Y \otimes_{\mathbb{Z}} k$ for any edge $E$ and if for any distinct edges $E$ and $E^{\prime}$ containing a common vertex we have $k \alpha_{E} \cap k \alpha_{E^{\prime}}=0$.

Note that this can be considered, for given $\mathcal{G}$, as a requirement on the characteristic of $k$. The main result of [Fie10] is the following:

Theorem 6.9 ([Fie10, Theorem 5.1]). Suppose that $(\mathcal{G}, k)$ is a GKMpair and that $\mathcal{B}$ is self-dual of degree $2 l$. Then we have

$$
\Omega_{k}(\mathcal{G})=\left\{\begin{array}{l|l}
x \in \mathcal{V} & \begin{array}{l}
\text { for all } y \geq x \text { the number } \\
\text { of edges containing } y \text { is } l
\end{array}
\end{array}\right\} .
$$

We are going to apply this statement later in order to study the $k$-smooth locus of $T$-varieties. 
6.5. The combinatorics of parity sheaves. Let $X$ be a complex $T$ variety, $k$ a complete local principal ideal domain. Assume that these data satisfy the assumptions (A1)-(A4a/b), (S1), (S2) and (R1)-(R3). We now come to the principal result of this paper.

Theorem 6.10. Suppose that $\mathcal{P} \in D_{T}^{b}(X, k)$ is a parity sheaf. Then $\mathbb{W}(\mathcal{P})$ is a Braden-MacPherson sheaf. More precisely, $\mathbb{W}(\mathcal{P}(\lambda)) \cong$ $\mathscr{B}(\lambda)$.

Proof. We have to show that $\mathbb{W}(\mathcal{P})$ satisfies the four properties listed in Definition 6.3. If we translate this definition into our situation we see that we have to check the following:

(1) For each $x \in X^{T}$ the cohomology $\mathbb{H}_{\mathrm{T}}^{\bullet}\left(\mathcal{P}_{x}\right)$ is a graded free module over $S_{k}$.

(2) For each one-dimensional orbit $E$ that is contained in the stratum associated to the fixed point $x$, the map

$$
\mathbb{H}_{\mathrm{T}}^{\bullet}\left(\mathcal{P}_{x}\right) \rightarrow \mathbb{H}_{\mathrm{T}}^{\bullet}\left(\mathcal{P}_{E}\right)
$$

is surjective with kernel $\alpha(E) \mathbb{H}_{\mathrm{T}}^{\bullet}\left(\mathcal{P}_{x}\right)$.

(3) For each open union $X_{\mathcal{J}} \subset X$ of strata the restriction homomorphism

$$
\mathbb{H}_{\mathrm{T}}^{\bullet}(\mathcal{P}) \rightarrow \mathbb{H}_{\mathrm{T}}^{\bullet}\left(\mathcal{P}_{\mathcal{J}}\right)
$$

is surjective.

(4) For each $x \in X^{T}$ the homomorphism $\mathbb{H}_{\mathrm{T}}^{\bullet}(\mathcal{P}) \rightarrow \mathbb{H}_{\mathrm{T}}^{\bullet}\left(\mathcal{P}_{x}\right)$ is surjective.

Part (1) follows directly from the definition of a parity sheaf, the parts (2) and (3) are stated in Proposition 5.6. Part (4) follows from Theorem 5.7 and the fact $\mathcal{P}$ is the direct sum of shifted copies of $\mathcal{P}(\lambda)$ 's. The last statement follows, as $\mathcal{P}(\lambda)$ is indecomposable if and only if $\mathbb{W}(\mathscr{B}(\lambda))$ is, by Theorem 5.11 .

\section{The CASe of Schubert Varieties}

We now discuss a special and important case of the general theory developed in the previous section, namely the case of Schubert varieties in (Kac-Moody) flag varieties. For a detailed construction of these varieties in the Kac-Moody setting the reader is referred to [Kum02].

We fix some notation. Let $A$ be a generalised Cartan matrix of size $l$ and let $\mathfrak{g}=\mathfrak{g}(A)=\mathfrak{n}_{-} \oplus \mathfrak{h} \oplus \mathfrak{n}_{+}$denote the corresponding Kac-Moody Lie algebra with Weyl group $W$, Bruhat order $\leq$, length function $\ell$ and simple reflections $S=\left\{s_{i}\right\}_{i=1, \ldots, l}$. To $A$ one may also associate a KacMoody group $G$ with Borel subgroup $B$ and connected algebraic torus $T \subset B$. Given any subset $I \subset\{1, \ldots, l\}$ one has a standard parabolic 
subgroup $P_{I}$ containing $B$ and standard parabolic subgroup $W_{I} \subset W$. The set $G / P_{I}$ may be given the structure of an ind- $T$-variety and is called a Kac-Moody flag variety.

For each $w \in W$ one may consider the Schubert cell $X_{w}^{I}:=B w P_{I} / P_{I} \subset$ $G / P_{I}$ and its closure, the Schubert variety,

$$
\overline{X_{w}^{I}}=\bigsqcup_{v \leq w} B v P_{I}
$$

Each Schubert cell is isomorphic to a (finite dimensional) affine space and each Schubert variety is a (finite dimensional) projective algebraic variety. The partition of $G / P_{I}$ into Schubert cells gives a stratification of $G / P_{I}$.

The following proposition shows that the results of this article may be applied to any closed union of finitely many $B$-orbits in $G / P_{I}$ :

Proposition 7.1. Let $X \subset G / P_{I}$ be a closed subset which is the union of finitely many Schubert cells. Then $X$ together with its stratification into Schubert cells satisfies our assumptions (A1), (A2), (S1), (S2), (R1), (R2) and (R3).

Proof. The assumptions (A1), (A2), (S1) are standard properties of Kac-Moody Schubert varieties (see [Kum02, Chapter 7]) and (S2) follows because we have an equivalence $D_{T, \Lambda}^{b}(X, k) \cong D_{B}^{b}(X, k)$. Given a Schubert variety $\overline{X_{w}^{I}} \subset X$, let $\pi: \widetilde{X} \rightarrow \overline{X_{w}^{I}}$ denote a Bott-Samelson resolution (see [Kum02, 7.1.3]). Then $\tilde{X}$ is a smooth $T$-variety with finitely many $T$-fixed points which admits a $T$-equivariant closed linear embedding into a projective space. Lastly, the variety $\widetilde{X}$ is even a $B$-variety, and the map $\pi$ is $B$-equivariant. So properties (R1), (R2) and (R3) hold as well.

We now describe the moment graph of $G / P_{I}$. The identification of $\mathfrak{h}$ with the Lie algebra of $T$ allows us to identify the lattice of characters $X^{*}(T)$ with a lattice in $\mathfrak{h}^{*}$. Moreover, under this identification, all the roots of $\mathfrak{g}(A)$ lie in $X^{*}(T)$. Let $R \subset X^{*}(T)$ denote the subset of real roots, and $R^{+}$the subset of real positive roots. Then we have a bijection

$$
\begin{array}{rcc}
R^{+} \stackrel{\sim}{\rightarrow} & \text { rreflections in } W\} \\
\alpha \mapsto & s_{\alpha} .
\end{array}
$$

The following proposition follows from [Kum02, Chapter 7]:

Proposition 7.2. We have: 
- The T-fixed points are in bijection with the set $W / W_{I}$ :

$$
\begin{gathered}
W / W_{I} \stackrel{\sim}{\rightarrow}\left(G / P_{I}\right)^{T} \\
w W_{I} \mapsto w P_{I} .
\end{gathered}
$$

- There is a one-dimensional T-orbit with $x W_{I}$ and $y W_{I}$ in its closure if and only if $s_{\alpha} x W_{I}=y W_{I}$ for some reflection $s_{\alpha} \in W$ in which case $T$ acts on this orbit with character $\pm \alpha$.

We complete this section by discussing what the arithmetic assumptions (A3), (A4a) and (A4b) mean in the case of Kac-Moody flag varieties. First note that the lattice $\mathbb{Z} R \subset X^{*}(T)$ spanned by the real roots determines a surjection of algebraic tori

$$
s: T \rightarrow T^{\prime}
$$

so that $X^{*}\left(T^{\prime}\right)=\mathbb{Z} R$. The action of $T$ on $G / P_{I}$ is trivial on the kernel of $s$ and we obtain an action of $T^{\prime}$ on $G / P_{I}$. (In the case of a finite dimensional Schubert variety this corresponds to the fact that one may always choose the adjoint form of a reductive group in order to construct the flag variety.) Because real roots are never divisible in $\mathbb{Z} R=X^{*}\left(T^{\prime}\right)$ it follows that (A4b) (and hence (A3)) is always satisfied for Kac-Moody Schubert varieties viewed as $T^{\prime}$-varieties.

The condition (A3) is more subtle. If we fix a field $k$ then condition (A3) is satisfied if and only if no two distinct roots in $R^{+}$become linearly dependent modulo $k$. One may check that in the finite cases we have to exclude characteristic 2 in non-simply laced types and characteristic 3 in type $G_{2}$.

In the affine case the situation is radically different. Suppose that $\widehat{G}$ is the affine Kac-Moody group associated to a semi-simple group $G$. Recall that there exists a an element $\delta \in \widehat{\mathfrak{h}}^{*}$ such that the set of real roots of $\widehat{G}$ is equal to $\{\alpha+n \delta\}$ where $\alpha \in \mathfrak{h}^{*}$ is a root of $G$, and $n \in \mathbb{Z}$. It follows that condition (A3) is satisfied for $\widehat{G} / \widehat{P}$ and any parabolic subgroup $\widehat{P} \neq \widehat{G}$ if and only if $k$ is of characteristic 0 .

However, if one restricts oneself to a fixed a Schubert variety $X \subset$ $\widehat{G} / \widehat{P}$ the GKM-condition for $X$ may yield interesting restrictions on the characteristic of $k$ (see [Fie07a]).

\section{8. $p$-SMOOTHNESS}

In this section we recall the definition and basic properties of the $p$-smooth locus of a complex algebraic variety $X$. Our main goal is Theorem 8.8 for which we need Proposition 8.6, where we show that an (a priori weaker) condition on the stalks of the intersection cohomology complex is enough to conclude $p$-smoothness. 
Throughout this section all varieties will be irreducible and $k$ denotes a ring (assumed to be a field from Sections 8.2 to 8.4). Dimension will always refer to the complex dimension. Given a point $y$ in a variety $Y$ we denote by $i_{y}:\{y\} \hookrightarrow Y$ its inclusion.

8.1. Smoothness and $p$-smoothness. If $x$ is a smooth point of a variety $X$ of dimension $n$ a simple calculation (using the long exact sequence of cohomology, excision and the cohomology of a $2 n-1$ sphere) yields

$$
H^{d}(X, X \backslash\{x\}, k)= \begin{cases}k, & \text { if } d=2 \operatorname{dim} X \\ 0, & \text { otherwise }\end{cases}
$$

The isomorphism

$$
H^{d}\left(i_{x} \underline{k}_{X}\right) \cong H^{d}(X, X \backslash\{x\}, k)
$$

motivates the following.

Definition 8.1. A variety $X$ is $k$-smooth if, for all $x \in X$, one has an isomorphism

$$
H^{d}\left(i_{x} \underline{k}_{X}\right) \cong \begin{cases}k, & \text { if } d=2 \operatorname{dim} X, \\ 0, & \text { otherwise. }\end{cases}
$$

The $k$-smooth locus of $X$ is the largest open $k$-smooth subvariety of $X$. We define $p$-smooth (respectively the $p$-smooth locus) to mean $\mathbb{F}_{p^{-}}$ smooth (respectively the $\mathbb{F}_{p}$-smooth locus).

Proposition 8.2. We have inclusions

$$
\begin{gathered}
\mathbb{Q} \text {-smooth } \\
\text { locus }
\end{gathered} \supset \begin{gathered}
\text { p-smooth } \\
\text { locus }
\end{gathered} \supset \begin{gathered}
\mathbb{Z} \text {-smooth } \\
\text { locus }
\end{gathered} \supset \begin{gathered}
\text { smooth } \\
\text { locus }
\end{gathered} .
$$

Proof. The fact that the $\mathbb{Z}$-smooth locus contains the smooth locus follows from the above discussion. For all rings $k$ one has an isomorphism

$$
i_{x}^{!} \underline{k}_{X} \cong i_{x}^{!} \underline{\mathbb{Z}}_{X} \stackrel{L}{\otimes_{\mathbb{Z}}} k
$$

As the category of $\mathbb{Z}$-modules is hereditary, every object in $D^{b}(\{x\}, \mathbb{Z})$ is isomorphic to its cohomology. It then follows from (6) that, for a field $k$, the condition of $k$-smoothness is satisfied at $x$ if and only if:

(1) $H^{d}\left(i_{x} \underline{\mathbb{Z}}_{X}\right)$ is torsion except for $d=2 n$, where the free part is of rank 1 ,

(2) all torsion in $H^{\bullet}\left(i_{x}^{!} \underline{\mathbb{Z}}_{X}\right)$ is prime to the characteristic of $k$. The claimed inclusions now follow easily.

Remark 2. The above proof shows that, if $k$ is a field, then the $k$-smooth locus only depends on the characteristic of $k$. 
8.2. $k$-smoothness and the intersection cohomology complex. Until Section 8.4 we assume that $k$ is a field. Let us denote by $\left(D^{\leq 0}(X, k), D^{\geq 0}(X, k)\right)$ the standard $t$-structure on $D(X, k)$ with heart $S h(X, k)$, the abelian category of sheaves of $k$-vector spaces on $X$. We denote the corresponding truncation and cohomology functors by $\tau_{\leq 0}, \tau_{>0}$ and $\mathcal{H}^{d}$.

Let us a fix a Whitney stratification $X=\bigsqcup_{\lambda \in \Lambda} X_{\lambda}$ and denote for all $\lambda \in \Lambda$ by $i_{\lambda}: X_{\lambda} \hookrightarrow X$ the inclusion. Recall that the intersection cohomology complex of $X, \mathbf{I C}(X, k) \in D(X, k)$, is uniquely determined by the properties:

(1) $i_{\lambda}^{*} \mathbf{I C}(X, k) \cong \underline{k}_{X_{\lambda}}$ for the open stratum $X_{\lambda} \subset X$;

and, for all strata $X_{\lambda}$ of strictly positive codimension,

(2) $\mathcal{H}^{d}\left(i_{\lambda}^{*} \mathbf{I C}(X, k)\right)=0$ for $d \geq \operatorname{codim}_{X} X_{\lambda}$,

(3) $\mathcal{H}^{d}\left(i_{\lambda}^{!} \mathbf{I C}(X, k)\right)=0$ for $d \leq \operatorname{codim}_{X} X_{\lambda}$.

Note that under this normalisation $\mathbf{I C}(X, k)$ is not Verdier self-dual. Rather $\mathbb{D} \mathbf{I C}(X, k) \cong \mathbf{I C}(X, k)[2 \operatorname{dim} X]$. Conditions (2) and (3) are equivalent to the conditions

$\left(2^{*}\right) \mathcal{H}^{d}\left(i_{x}^{*} \mathbf{I C}(X, k)\right)=0$ for $d \geq \operatorname{codim}_{X} X_{\lambda}$,

$\left(3^{*}\right) \mathcal{H}^{d}\left(i_{x}^{!} \mathbf{I C}(X, k)\right)=0$ for $d \leq \operatorname{dim} X+\operatorname{dim} X_{\lambda}$

for all $x \in X_{\lambda}$ and strata $X_{\lambda}$ of strictly positive codimension. (This follows from the fact if $y \in Y$ is a smooth point, then one has an isomorphism $i_{y}^{!} \underline{k}_{X_{\lambda}} \cong i_{y}^{*} \underline{k}_{X_{\lambda}}[-2 \operatorname{dim} Y]$.)

Proposition 8.3. A variety $X$ is $k$-smooth if and only if $\mathbf{I C}(X, k) \cong$ $\underline{k}_{X}$.

Proof. If $X$ is $k$-smooth then the constant sheaf $\underline{k}_{X}$ satisfies (1), (2*) and $\left(3^{*}\right)$ above and hence $\underline{k}_{X} \cong \mathbf{I C}(X, k)$. On the other hand, if $\underline{k}_{X} \cong \mathbf{I C}(X, k)$ then $\mathbb{D} \underline{k}_{X} \cong \underline{k}_{X}[2 \operatorname{dim} X]$ and for all $x \in X$ we have

$$
i_{x}^{!} \underline{k}_{X} \cong i_{x}^{!}\left(\mathbb{D} \underline{k}_{X}\right)[-2 \operatorname{dim} X] \cong \mathbb{D}\left(i_{x}^{*} \underline{k}_{X}[2 \operatorname{dim} X]\right)
$$

and hence

$$
H^{d}\left(i_{x}^{!} \underline{k}_{X}\right)=H^{-d}\left(i_{x}^{*} \underline{k}_{X}[2 \operatorname{dim} X]\right)= \begin{cases}k, & \text { if } d=2 \operatorname{dim} X \\ 0, & \text { otherwise }\end{cases}
$$

and so $x$ is $k$-smooth.

8.3. $k$-smoothness and stalks. Given a morphism $f: X \rightarrow Y$ of complex algebraic varieties we write ${ }^{0} f_{*}$ for the non-derived direct image functor. The functor ${ }^{0} f_{*}$ is left $t$-exact with respect to the standard $t$-structure. Given $\mathcal{F} \in S h(X, k)$ we have ${ }^{0} f_{*} \mathcal{F} \cong \tau_{\leq 0} f_{*} \mathcal{F}$ canonically.

Lemma 8.4. Given $\mathcal{F} \in D^{\geq 0}(X, k)$ and a morphism $f: X \rightarrow Y$ we have a natural isomorphism $\tau_{\leq 0} f_{*} \mathcal{F} \cong 0 f_{*} \tau_{\leq 0} \mathcal{F}$. 
Proof. Applying $f$ to the distinguished triangle

$$
\tau_{\leq 0} \mathcal{F} \rightarrow \mathcal{F} \rightarrow \tau_{>0} \mathcal{F} \rightarrow
$$

yields a distinguished triangle

$$
f_{*} \tau_{\leq 0} \mathcal{F} \rightarrow f_{*} \mathcal{F} \rightarrow f_{*} \tau_{>0} \mathcal{F} \rightarrow
$$

Now $f_{*}$ is left $t$-exact for the $t$-structure $\left(D^{\leq 0}(X, k), D^{\geq 0}(X, k)\right)$ and so $\tau_{\leq 0} f_{*} \tau_{>0} \mathcal{F}=0$. Hence if we apply $\tau_{\leq 0}$ to the above distinguished triangle we obtain the required isomorphism

$$
{ }^{0} f_{*} \tau_{\leq 0} \mathcal{F}=\tau_{\leq 0} f_{*} \tau_{\leq 0} \mathcal{F} \stackrel{\sim}{\rightarrow} \tau_{\leq 0} f_{*} \mathcal{F} .
$$

Lemma 8.5. We have an isomorphism $\tau_{\leq 0} \mathbf{I C}(X, k) \cong{ }^{0} j_{*} \underline{k}_{U}$, where $j: U \hookrightarrow X$ denotes the inclusion of a smooth, open, dense subvariety of $X$.

Proof. Choose a stratification of $X$ which has $U$ as the only stratum of dimension $n$, and write $X_{i}$ for the union of all strata of codimension $\leq i$ (so that $X_{0}=U$ and $\left.X_{n}=X\right)$. We have a chain of inclusions

$$
X_{0} \stackrel{j_{0}}{\hookrightarrow} X_{1} \stackrel{j_{1}}{\hookrightarrow} X_{2} \stackrel{j_{0}}{\longrightarrow} \cdots \stackrel{j_{n-2}}{\longrightarrow} X_{n-1} \stackrel{j_{n-1}}{\longrightarrow} X_{n}
$$

The Deligne construction (see [BBD82, Proposition 2.1.11]) gives an isomorphism

$$
\mathbf{I C}(X, k) \cong\left(\tau_{\leq n-1} \circ j_{n-1 *}\right) \circ\left(\tau_{\leq n-1} \circ j_{n-1 *}\right) \circ \ldots\left(\tau_{\leq 0} \circ j_{0 *} \underline{k}_{U}\right)
$$

Repeatedly applying the above lemma yields

$$
\tau_{\leq 0} \mathbf{I C}(X, k) \cong{ }^{0} j_{n-1 *} \circ \cdots \circ{ }^{0} j_{1 *} \circ{ }^{0} j_{0 *} \underline{k}_{U} \cong{ }^{0} j_{*} \underline{k}_{U},
$$

as claimed.

Proposition 8.6. A variety $X$ is $k$-smooth if and only if $\mathbf{I C}(X, k)_{x} \cong$ $k$ for all $x \in X$.

Proof. If $X$ is $k$-smooth then $\mathbf{I C}(X, k) \cong \underline{k}_{X}$ by Proposition 8.3 and so $\mathbf{I C}(X, k)_{x} \cong k$ for all $x \in X$. It remains to show the converse. Choose an open, dense, smooth subvariety $U$ of $X$ and let $j: U \hookrightarrow X$ denote its inclusion. The adjunction morphism

$$
\underline{k}_{X} \rightarrow{ }^{0} j_{*} j^{*} \underline{k}_{X}
$$

is an injection on stalks, as may easily be checked from the definition of ${ }^{0} j_{*}$. (It is an isomorphism if and only if $X$ is unibranched, however we will not need this fact.) It follows from our assumptions that $\mathbf{I C}(X, k)$ lies in $D^{\leq 0}(X, k)$ and so we have an isomorphism

$$
\tau_{\leq 0} \mathbf{I C}(X, k) \stackrel{\sim}{\rightarrow} \mathbf{I C}(X, k) .
$$


By the above lemma we also have an isomorphism ${ }^{0} j_{*} \underline{k}_{U} \cong \tau_{\leq 0} \mathbf{I C}(X, k)$. It follows that all stalks of ${ }^{0} j_{*} \underline{k}_{U}$ are one-dimensional and that we have an isomorphism

$$
\underline{k}_{X} \stackrel{\sim}{\rightarrow}{ }^{0} j_{*} \underline{k}_{U} \stackrel{\sim}{\rightarrow} \mathbf{I C}(X, k) .
$$

Our claim now follows from Proposition 8.3.

8.4. On the $p$-smooth locus of $T$-varieties. Now let $X=\bigsqcup_{\lambda \in \Lambda} X_{\lambda}$ be an irreducible, complex, stratified $T$-variety, and let $k$ be a field. Assume that these data satisfy the assumptions (A1)-(A4a/b), (S1), $(\mathrm{S} 2)$ and $(\mathrm{R} 1)-(\mathrm{R} 3)$ and let $\Omega_{k}(\mathcal{G})$ denote the $k$-smooth locus of the moment graph $\mathcal{G}$ of $X$. The following proposition shows that the $k$ smooth locus of $X$ and of its moment graph agree.

Proposition 8.7. All points of a stratum $X_{\lambda}$ belong to the $k$-smooth locus of $X$ if and only if $\lambda \in \Omega_{k}(\mathcal{G})$.

Proof. Let $U$ denote the $p$-smooth locus of $X$. It is a union of strata by our assumption (S2). Because we have assumed that $X$ is irreducible there exists a unique open dense stratum $X_{\lambda} \subset X$. Let $\mathcal{P}$ be the corresponding indecomposable parity sheaf normalised so that its restriction to $X_{\lambda}$ is $\underline{k}_{X_{\lambda}}$.

In the following it will be useful to work with non-equivariant sheaves. Note that the non-equivariant analogue of Theorem 5.3 is valid (see [JMW09a, Theorem 2.12]) and $\overline{\mathcal{P}}:=\operatorname{For}(\mathcal{P})$ is the indecomposable non-equivariant parity sheaf with support $X$.

Let $U^{\prime}$ denote the largest open union of strata $X_{\lambda}$ for which $\overline{\mathcal{P}}_{X_{\lambda}} \cong$ $\underline{k}_{X_{\lambda}}$. We claim $U=U^{\prime}$.

Indeed, if $U^{\prime}$ denotes this set then $\overline{\mathcal{P}}_{U^{\prime}}$ satisfies the properties (1) and (2) of the IC-complexes and hence also satisfies (3) because $\mathbb{D}\left(\overline{\mathcal{P}}_{U^{\prime}}\right) \cong$ $\overline{\mathcal{P}}_{U^{\prime}}[2 \operatorname{dim} X]$. Hence $\overline{\mathcal{P}}_{U^{\prime}} \cong \mathbf{I C}(U, k)$ and so $U^{\prime} \subset U$ by Propositions 8.3 and 8.6. On the other hand, $\mathbf{I C}(U, k) \cong \underline{k}_{U}$ is certainly indecomposable and $*$-even. It is even !-even because $\mathbb{D} \mathbf{I C}(U, k) \cong \mathbf{I C}(U, k)[2 \operatorname{dim} X]$. Hence $\mathcal{P}_{U} \cong \underline{k}_{U}$ by the classification of parity sheaves, together with the fact that the restriction of an indecomposable parity sheaf to an open union of strata is either zero or indecomposable (see [JMW09a, Proposition 2.11]).

Now, by Theorem 6.10, $\mathbb{W}(\mathcal{P}) \cong \mathscr{B}(\lambda)$ and hence $\overline{\mathcal{P}}_{X_{\mu}} \cong \underline{k}_{X_{\lambda}}$ if and only if $\mathscr{B}(\lambda)^{\mu} \cong S_{k}$. The proposition then follows by definition of the $k$-smooth locus of the moment graph of $X$.

Combining this result with Theorem 6.9 yields: 
Theorem 8.8. A T-fixed point $x_{\mu} \in X_{\mu}$ belongs to the $p$-smooth locus of $X$ if and only if for all $\lambda \geq \mu$ the number of one-dimensional $T$ orbits having $x_{\lambda}$ in their closure is equal to the complex dimension of $X$. Moreover, $X_{\mu}$ belongs to the $k$-smooth locus if and only if its $T$-fixed point $x_{\mu}$ does.

8.5. A freeness result. In this subsection $k$ denotes a complete local ring and $p$ denotes the characteristic of the residue field of $k$. Let $X=\bigsqcup_{\lambda \in \Lambda} X_{\lambda}$ be an irreducible, complex, stratified $T$-variety. Assume that these data satisfy the assumptions (A1)-(A4a/b), (S1), (S2). We further assume that there exists an indecomposable parity sheaf $\mathcal{P}$ corresponding to the unique open stratum $X_{\mu} \subset X$. (For example, $X$ could be open in a stratified variety satisfying (R1), (R2) and (R3).) For any $\lambda \in \Lambda$ let

$$
X_{>\lambda}=\bigsqcup_{\gamma>\lambda} X_{\gamma} \quad \text { and } \quad X_{\geq \lambda}=\bigsqcup_{\gamma \geq \lambda} X_{\gamma}
$$

For any $\lambda \in \Lambda$ we can find a $T$-stable affine neighbourhood $U$ of $x_{\lambda}$ and a $T$-invariant affine normal slice $N \subset U$ to the stratum $X_{\lambda}$. The aim of this section is to show the following result:

Proposition 8.9. $\mathbb{H}_{\mathrm{T}}^{\bullet}\left(\mathcal{P}_{N \backslash\left\{x_{\lambda}\right\}}\right)$ is torsion free over $k$.

Of course this result has no content if $k$ is a field. However it seems to be quite useful if $k$ is, for example, the $p$-adic integers. Before turning to the proof of this result we state a corollary, which is of central importance to $[\mathrm{JW}]$ :

Corollary 8.10. If $X_{>\lambda}$ is $p$-smooth then $H_{T}^{\bullet}\left(N \backslash\left\{x_{\lambda}\right\}, k\right)$ is a free $k$-module.

Proof. If $X_{>\lambda}$ is $p$-smooth then the constant sheaf with coefficients in $k$ is self-dual and hence parity. Hence the restriction of $\mathcal{P}$ to $X_{>\lambda}$ is isomorphic to the constant sheaf (cf. [JMW09a, Proposition 2.11]). The result then follows from Proposition 8.9.

Proof of Proposition 8.9. Consider the Cartesian diagram:

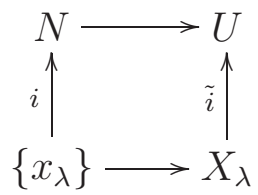

Without loss of generality we may assume that $X_{\lambda}$ is a closed stratum in $X$. In this case we have seen in the course of the proof of Theorem 5.11 
that we have an isomorphism $\mathbb{H}_{\mathrm{T}}^{\bullet}(\tilde{i} ! \mathcal{P}) \cong \mathbb{W}(\mathcal{P})_{\lambda}$. Moreover, because $N \hookrightarrow U$ is a normally non-singular inclusion we have

$$
\mathbb{H}_{\mathrm{T}}^{\bullet}\left(i^{!} \mathcal{P}_{N}\right)=\mathbb{H}_{\mathrm{T}}^{\bullet}(\tilde{i} ! \mathcal{P}) \cong \mathbb{W}(\mathcal{P})_{\lambda}
$$

One the other hand, by the attractive proposition, we have $\mathbb{H}_{\mathrm{T}}^{\bullet}\left(\mathcal{P}_{N}\right)=$ $\mathbb{H}_{\mathrm{T}}^{\bullet}\left(\mathcal{P}_{x_{\lambda}}\right)=\mathbb{W}(\mathcal{P})^{\lambda}$.

Now consider the open-closed decomposition:

$$
\left\{x_{\lambda}\right\} \stackrel{i}{\hookrightarrow} N \stackrel{j}{\hookleftarrow} N \backslash\left\{x_{\lambda}\right\} .
$$

This leads to a distinguished triangle

$$
i_{i} i \cdot \mathcal{P} \rightarrow \mathcal{P}_{U} \rightarrow j_{*} j^{*} \mathcal{P} \stackrel{[1]}{\rightarrow}
$$

Taking hypercohomology and using the above observations we conclude that we have an exact sequence

$$
0 \rightarrow \mathbb{W}(\mathcal{P})_{\lambda} \rightarrow \mathbb{W}(\mathcal{P})^{\lambda} \rightarrow \mathbb{H}_{\mathrm{T}}^{\bullet}\left(\mathcal{P}_{N \backslash\left\{x_{\lambda}\right\}}\right) \rightarrow 0
$$

where the first map is the inclusion. It follows that we have an embedding

$$
\mathbb{H}_{\mathrm{T}}^{\bullet}\left(\mathcal{P}_{N \backslash\left\{x_{\lambda}\right\}}\right) \hookrightarrow \bigoplus_{E: \lambda \rightarrow \gamma} \mathbb{W}(\mathcal{P})_{E}
$$

Now each $\mathbb{W}(\mathcal{P})_{E}$ is isomorphic to a direct sum of shifts of $S /\left(\alpha_{E}\right)$. By assumption (A4b) no character $\alpha_{E}$ is $p$-divisible in $X(T)$ and hence each $S /\left(\alpha_{E}\right)$ is torsion free over $k$. It follows that $\mathbb{H}_{\mathrm{T}}^{\bullet}\left(\mathcal{P}_{N \backslash\left\{x_{\lambda}\right\}}\right)$ is torsion free over $k$.

\section{RePRESENTATIONS OF REDUCTIVE ALGEBRAIC GROUPS}

Let $G$ be a simple reductive algebraic group over $\overline{\mathbb{F}}_{p}$ and let $\operatorname{Rep} G$ denote the category of rational representations of $G$. It is a fundamental problem in representation theory to determine the characters of the simple and tilting modules in $\operatorname{Rep} G$. For simple modules there exists a conjecture, due to Lusztig, in the case that the characteristic $p$ is larger than the Coxeter number $h$ associated to $G$. For tilting modules there is no general conjecture. Schur-Weyl duality can be used to show that knowledge of the characters of tilting modules for $G=G L_{n}\left(\overline{\mathbb{F}}_{p}\right)$ implies dimension formula for the simple modules for $S_{m}$ for $m \leq n$ in characteristic $p$.

We want to explain how the above results allow one to reinterpret these two basic problems using the geometry of certain Schubert varieties in the (complex) affine Grassmannian associated with the Langlands dual group. To this end let $T \subset B \subset G$ denote a maximal torus 
and Borel subgroup of $G$ respectively. Let $X^{*}(T)$ denote the character lattice and $X^{+}(T)$ denote the subset of dominant weights. Then $X^{+}(T)$ parametrises both the simple and tilting modules in $\operatorname{Rep} G$.

9.1. Tilting modules. Let $G_{\mathbb{C}}^{\vee}$ denote the complex Langlands dual group of $G, G_{\mathbb{C}}^{\vee}((t))$ its loop group, $\widehat{T_{\mathbb{C}}^{\vee}}=T_{\mathbb{C}}^{\vee} \times \mathbb{C}^{\times}$the extended torus and $\mathcal{G} \mathrm{r}^{\vee}:=G_{\mathbb{C}}^{\vee}((t)) / G_{\mathbb{C}}^{\vee}[[t]]$ the corresponding affine Grassmannian. Then $X^{+}(T)$ also parametrises the $G_{\mathbb{C}}^{\vee}[[t]]$-orbits on $\mathcal{G r}^{\vee}$ and $\mathcal{G}^{\vee}$ satisfies our assumptions when viewed with the action of $\widehat{T_{\mathbb{C}}^{V}}$ (indeed, the closures of $G_{\mathbb{C}}^{\vee}[[t]]$-orbits are examples of Kac-Moody Schubert varieties). Recall that the geometric Satake equivalence [MV07] establishes a tensor equivalence between the abelian category of rational representations of $G$ and the tensor category of $G_{\mathbb{C}}^{\vee}[[t]]$-equivariant perverse sheaves on $\mathcal{G r}^{\vee}$.

Recall the following two results which are Theorem 5.1 and Corollary 5.8 of [JMW09b]:

(1) If $p>h+1$, then parity sheaves correspond under the geometric Satake isomorphism to tilting modules. More precisely, the indecomposable parity sheaf $\mathcal{P}(\lambda)$ corresponds to the indecomposable tilting module $T(\lambda)$.

(2) The rank of $\mathbb{H}_{\mathrm{T}}^{\bullet}\left(\mathcal{P}(\lambda)_{\mu}\right)$ is equal to the dimension of the $\mu$-weight space of the tilting module $T(\lambda)$.

With the above results in mind, it seems natural to expect that the Braden-MacPherson algorithm can be used to calculate the characters of tilting modules. There is a problem, however: the moment graph of the affine Grassmannian satisfies the GKM-condition if and only if $k$ is of characteristic 0 .

To get around this problem we take $k$ to be the ring $\mathbb{Z}_{p}$ of $p$-adic numbers. For this the GKM-condition is satisfied. Moreover it is shown [JMW09a, Proposition 2.41] that the graded ranks of the stalks of parity sheaves depend only on the characterstic of the residue field. The following theorem then follows from the above discussion and our main theorem:

Theorem 9.1. Suppose that $p>h+1$ (see [JMW09b] for better bounds). When conducted with coefficients in the ring $k=\mathbb{Z}_{p}$ of p-adic numbers, the Braden-MacPhersons algorithm computes the characters of tilting modules. More precisely, for any character $\mu \in X^{*}(T)$, the dimension of the $\mu$-weight space of $T(\lambda)$ is equal to the rank of $\mathscr{B}(\lambda)^{\mu}$.

9.2. Simple rational characters. We now turn to the application of the above results to Lusztig's conjecture. Let $I^{\vee} \subset G_{\mathbb{C}}^{\vee}((t))$ be the 
Iwahori subgroup containing $B^{\vee}$ and let $\mathcal{F} 1^{\vee}:=G_{\mathbb{C}}^{\vee}((t)) / I^{\vee}$ denote the affine flag variety with its $\widehat{T_{\mathbb{C}}^{\vee}}$-action.

In $\left[\right.$ Fie07b] a certain subcategory $\mathcal{I} \subset D_{\widehat{T}}^{b}\left(\mathcal{F} \mathrm{l}^{\vee}, k\right)$ of special equivariant sheaves was considered and a functor $\Phi: \mathcal{I} \rightarrow \mathcal{R}$ was defined, where $\mathcal{R}$ is a category of projective objects in a category $\mathcal{C}$ naturally associated to the Lie algebra of $G$. For the application to Lusztig's conjecture one needs to consider only objects in $\mathcal{I}$ that are supported on a certain Schubert variety $X_{\text {res }} \subset \mathcal{F} \mathrm{l}^{\vee}$.

An intermediate step in the construction of $\Phi$ was a functor from $\mathcal{I}$ to the category of Braden-MacPherson sheaves on the moment graph associated to $\mathcal{F} l^{\vee}$. It turns out that $\mathcal{I}$ is the category of parity sheaves on $\mathcal{F} l^{\vee}$ (with respect to the stratification by Schubert cells). Indeed, the category $\mathcal{I}$ is generated from the skyscraper sheaf on the point stratum on $\mathcal{F} \mathrm{l}^{\vee}$ by repeatedly applying the functors $\pi_{s}^{*} \pi_{s *}$ for simple reflections $s$, where $\pi_{s}: \mathcal{F} l^{\vee} \rightarrow \mathcal{F} l_{s}^{\vee}$ is the projection onto the partial affine flag variety associated to $s$. Now parity sheaves are preserved by these functors (cf. [JMW09a, Proposition 4.9]). From the results in $[$ Fie07b] we can hence deduce that the ranks of the stalks of parity sheaves determine baby Verma multiplicities for projective objects in $\mathcal{C}$. These multiplicities in turn determine the characters of simple rational representations of $G$. Using the results of this paper we can rephrase the above as follows. Given $\lambda \in \Lambda$ let $\mathbf{I C}\left(\bar{X}_{\lambda}, \mathbb{Z}\right)$ denote the intersection cohomology complex of $\bar{X}_{\lambda}$ with integral coefficients (cf. [Jut09]).

Theorem 9.2. Suppose that the stalks and costalks of the intersection cohomology complex $\mathbf{I C}\left(\overline{X_{\lambda}}, \mathbb{Z}\right)$ are $p$-torsion-free for all strata $X_{\lambda} \subset$ $X_{\text {res. }}$. Then the characters of the simple modules for $G$ are given by Lusztig's conjecture.

Proof. It is known (cf. [Lus83]) that $\operatorname{IC}\left(\bar{X}_{\lambda}, \mathbb{Z}\right) \stackrel{L}{\otimes} \mathbb{Q}$ is isomorphic to the parity sheaf $\mathcal{P}_{\mathbb{Q}}(\lambda)$ with coefficients in $\mathbb{Q}$. Hence $\mathbf{I C}\left(\bar{X}_{\lambda}, \mathbb{Z}\right) \stackrel{L}{\otimes} \mathbb{F}_{p}$ is isomorphic to the parity sheaf $\mathcal{P}_{\mathbb{F}_{p}}(\lambda)$ with coefficients in $\mathbb{F}_{p}$ if and only if the conditions of the theorem are met. The theorem then follows from [Fie07a, Fie07b] together with our main theorem.

\section{REFERENCES}

[Ara98] A. Arabia. Classes d'Euler équivariantes et points rationnellement lisses. (Equivariant Euler classes and rationally smooth points.). Ann. Inst. Fourier, 48(3):861-912, 1998.

[BB81] A. Beřlinson and J. Bernstein. Localisation de g-modules. C. R. Acad. Sci. Paris Sér. I Math., 292(1):15-18, 1981. 
[BBD82] A. Bẹlinson, J. Bernstein, and P. Deligne. Faisceaux pervers. In Analyse et topologie sur les espaces singuliers, I (Luminy, 1981), volume 100 of Astérisque, pages 5-171. Soc. Math. France, Paris, 1982.

[BL94] J. Bernstein and V. Lunts. Equivariant sheaves and functors, volume 1578 of Lecture Notes in Mathematics. Springer-Verlag, Berlin, 1994.

[BM01] T. Braden and R. MacPherson. From moment graphs to intersection cohomology. Math. Ann., 321(3):533-551, 2001.

[Bra03] T. Braden. Hyperbolic localization of intersection cohomology. Transform. Groups, 8(3):209-216, 2003.

[Bri98] M. Brion. Equivariant cohomology and equivariant intersection theory. In Representation theories and algebraic geometry (Montreal, $P Q$, 1997), volume 514 of NATO Adv. Sci. Inst. Ser. C Math. Phys. Sci., pages 1-37. Kluwer Acad. Publ., Dordrecht, 1998. Notes by Alvaro Rittatore.

[Car94] J. B. Carrell. The Bruhat graph of a Coxeter group, a conjecture of Deodhar, and rational smoothness of Schubert varieties. In Algebraic groups and their generalizations: classical methods (University Park, PA, 1991), volume 56 of Proc. Sympos. Pure Math., pages 53-61. Amer. Math. Soc., Providence, RI, 1994.

[CG83] J. Carrell and M. Goresky. A decomposition theorem for the integral homology of a variety. Invent. Math., 73:367-381, 1983.

[CS74] T. Chang and T. Skjelbred. The topological Schur lemma and related results. Ann. of Math. (2), 100:307-321, 1974.

[Dug66] J. Dugundji. Topology. Allyn and Bacon Inc., Boston, Mass., 1966.

[Dye93] M. Dyer. The nil Hecke ring and Deodhar's conjecture on Bruhat intervals. Invent. Math., 111(3):571-574, 1993.

[Dye05] M. Dyer. Rank two detection of singularities of Schubert varieties. preprint arXiv:0906.2994, 2005.

[Fie07a] P. Fiebig. Lusztig's conjecture as a moment graph problem. Bull. London Math. Soc. 42(6) (2010), 957-972.

[Fie07b] P. Fiebig. Sheaves on affine Schubert varieties, modular representations and Lusztig's conjecture. J. Amer. Math. Soc. 24 (2011), 133-181.

[Fie10] P. Fiebig. The multiplicity one case of Lusztig's conjecture. Duke Math. J., 153:551-571, 2010.

[Ful97] W. Fulton. Young tableaux. With applications to representation theory and geometry. London Mathematical Society Student Texts. 35. Cambridge: Cambridge University Press., 1997.

[GKM98] M. Goresky, R. Kottwitz, and R. MacPherson. Equivariant cohomology, Koszul duality, and the localization theorem. Invent. Math., 131(1):2583, 1998.

[Jan03] J. C. Jantzen. Representations of algebraic groups, volume 107 of Mathematical Surveys and Monographs. American Mathematical Society, Providence, RI, second edition, 2003.

[Jan09] J. C. Jantzen. Moment graphs and representations. Lecture notes, 33 pages, www-fourier.ujf-grenoble.fr/IMG/pdf/notes_jantzen-2. pdf, 2009.

[JMW09a] D. Juteau, C. Mautner, and G. Williamson. Parity sheaves. preprint arXiv:0906.2994, 2009. 
[JMW09b] D. Juteau, C. Mautner, and G. Williamson. Perverse sheaves and modular representation theory. preprint arXiv:0901.3322, 2009.

[JMW09b] D. Juteau, C. Mautner, and G. Williamson. Parity sheaves and tilting modules. in preparation, see arXiv:0901.3322v1.

$[\mathrm{JW}] \quad$ D. Juteau and G. Williamson. Kumar's criterion modulo $p$. preprint 2011.

[Jut09] D. Juteau. Decomposition numbers for perverse sheaves. Ann. Inst. Fourier, 59, 2009. To appear.

[KKLV89] F. Knop, H. Kraft, D. Luna, and T. Vust. Local properties of algebraic group actions. Algebraische Transformationsgruppen und Invariantentheorie, DMV Semin. 13, 63-75 (1989), 1989.

[KS94] M. Kashiwara and P. Schapira. Sheaves on manifolds, volume 292 of Grundlehren der Mathematischen Wissenschaften [Fundamental Principles of Mathematical Sciences]. Springer-Verlag, Berlin, 1994. With a chapter in French by Christian Houzel, Corrected reprint of the 1990 original.

[Kum96] S. Kumar. The nil Hecke ring and singularity of Schubert varieties. Invent. Math., 123(3):471-506, 1996.

[Kum02] S. Kumar. Kac-Moody groups, their flag varieties and representation theory, volume 204 of Progress in Mathematics. Birkhäuser Boston Inc., Boston, MA, 2002.

[Lus83] G. Lusztig. Singularities, character formulas, and a $q$-analog of weight multiplicities. In Analyse et topologie sur les espaces singuliers, II, III (Luminy, 1981), volume 101-102 of Astérisque, pages 208-229. Soc. Math. France, Paris, 1983.

[MS74] J. W. Milnor and J. D. Stasheff. Characteristic classes. Princeton University Press, Princeton, N. J., 1974. Annals of Mathematics Studies, No. 76.

[MV07] I. Mirković and K. Vilonen. Geometric Langlands duality and representations of algebraic groups over commutative rings. Ann. of Math. (2), 166(1):95-143, 2007.

[SHS69] Séminaire Heidelberg-Strasbourg, 1966/1967: Dualité de Poincaré. Publication I.R.M.A. Strasbourg, No. 3. Institut de Recherche Mathématique Avancée, Laboratoire Associé au C.N.R.S., Université de Strasbourg, Strasbourg, 1969.

[Soe00] W. Soergel. On the relation between intersection cohomology and representation theory in positive characteristic. J. Pure Appl. Algebra, 152(13):311-335, 2000.

[Spa88] N. Spaltenstein. Resolutions of unbounded complexes. Compos. Math., 65(2):121-154, 1988.

[Spr82] T. A. Springer. Quelques applications de la cohomologie d'intersection. In Bourbaki Seminar, Vol. 1981/1982, volume 92 of Astérisque, pages 249-273. Soc. Math. France, Paris, 1982.

[Spr84] T. A. Springer. A purity result for fixed point varieties in flag manifolds. J. Fac. Sci. Univ. Tokyo Sect. IA Math., 31(2):271-282, 1984.

[Sum74] H. Sumihiro. Equivariant completion. J. Math. Kyoto Univ., 14:1-28, 1974 . 